\title{
Characterizing Permissibility, Proper Rationalizability, and Iterated Admissibility by Incomplete Information
}

\author{
Shuige Liu
}

Received: date / Accepted: date

\begin{abstract}
We characterize three interrelated concepts in epistemic game theory: permissibility, proper rationalizability, and iterated admissibility. We define the lexicographic epistemic model for a game with incomplete information. Based on it, we give two groups of characterizations. The first group characterizes permissibility and proper rationalizability. The second group characterizes permissibility in an alternative way and iterated admissibility. In each group, the conditions for the latter are stronger than those for the former, which corresponds to the fact that proper rationalizability and iterated admissibility are two (compatible) refinements of permissibility within the complete information framework. The intrinsic difference between the two groups are the role of rationality: the first group does not need it, while the second group does
\end{abstract}

Keywords epistemic game theory · incomplete information · lexicographic belief . permissibility $\cdot$ proper rationalizability $\cdot$ iterated admissibility

\section{Introduction}

The purpose of noncooperative game theory is to study an individual's decision making in an interactive situation. Since there one's payoff is not completely determined

The author would like to express her gratitude to Andrés Perea for his thorough reading of the early versions of this paper and his numerous valuable comments and suggestions. She also would like to thank János Flesch, Funaki Yukihiko, Christian Bach, Abraham Neyman, Zsombor Z. Méder, and Dmitriy Kvasov for their valuable discussions and encouragements. She thanks all teachers and students in the 4th Epicenter Spring Course on Epistemic Game Theory in Maastricht University for their inspiring teaching and stimulating discussions. She gratefully acknowledge the support of Grant-in-Aids for Young Scientists (B) of JSPS No.17K13707 and Grant for Special Research Project No. 2017K-016 of Waseda University.

Shuige Liu

Faculty of Political Science and Economics, Waseda University, 1-6-1 Nishi-Waseda, Shinjuku-Ku, 1698050, Tokyo, Japan

E-mail: shuige_liu@aoni.waseda.jp 
by her own choice, decision making requires her to form a belief on every other participant's choice, on every other participant's belief on every other's choice, and so on. Studying the structure of those belief hierarchies and choices supported by a belief hierarchy satisfying some particular conditions opened up a field called epistemic game theory. See Perea (2002) for a textbook on this field.

In epistemic game theory, various concepts have been developed to describe specific belief structures. One is lexicographic belief (Blume et al. 1991a, 1991b). A lexicographic belief describes a player's subjective conjecture about the opponents' behavior by a sequence of probability distributions over other participants' choices and types, which is different from the adoption of a single probability distribution in a standard probabilistic belief. The interpretation of a lexicographic belief is that every choice-type pair in the sequence is considered to be possible, while a pair occurring ahead in the sequence is deemed infinitely more likely than one occurring later. Several concepts have been developed by putting various conditions on lexicographic beliefs intended to capture different styles of reasoning about the opponents' behavior. Permissibility, proper rationalizability, and iterated admissibility are three important and interrelated concepts among them.

Permissibility originated from Selten (1975)'s perfect equilibrium. It is defined and studied from the epistemic viewpoint by using lexicographic belief hierarchy in Brandenburger (1992) (an alternative approach without using lexicographic belief is given by Börgers, 1994). Permissibility is based on two notions: caution and primary belief in the opponents' rationality. A lexicographic belief is said to be cautious if it does not exclude any choice of the opponents; it is said to primarily believe in the opponents' rationality if its first level belief only deems possible those choice-type pairs where the choice is optimal under the belief of the paired type (Perea, 2012).

Proper rationalizability originated from Myerson (1978)'s proper equilibrium which is intended to be a refinement of perfect equilibrium. It is defined and studied in Schuhmacher (1999) and Asheim (2001) as an epistemic concept. Proper rationalizability shares with permissibility the notion of caution, while, instead of primary belief in the opponents' rationality, it is based on a stronger notion called respecting the opponents' preferences which means that a "better" choice always occurs in front of a "worse" choice in a lexicographic belief.

Iterated admissibility originated from the study of iteratively undominated choices (Bernheim, 1984, Pearce, 1984, Samuelson, 1992). In Brandenburger et al. (2008) and Perea (2012) it is explored as an epistemic concept by using lexicographic belief hierarchy. It is based on caution and a notion called assumption of rationality, which is also stronger than primary belief in the opponents' rationality. A lexicographic belief is said to assume the opponents' rationality if every "good" choice occurs and is always located in front of any "bad" one. Here good means that a choice of the opponent can be supported by a cautious belief of her, and bad means the opposite.

We illustrate the three concepts by two examples.

Example1,1 (Permissibility and proper rationalizability) Consider a game where player 1 has strategies $A$ and $B$ and player 2 has strategies $C, D$, and $E$. Player 2's utility function $u_{2}$ is illustrated in Table 1. Consider a lexicographic belief of player 1 on player 2's choices. Caution requires that all three choices of player 2 occur in 
Table 1 A two-person game for permissibility and proper rationalizability

\begin{tabular}{cccc}
\hline$u_{2}$ & $C$ & $D$ & $E$ \\
\hline$A$ & 3 & 2 & 1 \\
$B$ & 3 & 2 & 1
\end{tabular}

Table 2 A two-person game for iterated admissibility

\begin{tabular}{cccc}
\hline$u_{2}$ & $C$ & $D$ & $E$ \\
\hline$A$ & 3 & 2 & 1 \\
$B$ & 3 & 2 & $\underline{4}$ \\
\hline
\end{tabular}

that belief. Since $C$ is player 2's most preferred choice, primary belief in player 2's rationality requires that only choice $C$ can be put in the first level. Further, since player 2 prefers $C$ to $D$ and $D$ to $E$, a lexicographic belief of player 1 respecting 2's preferences should deem $C$ infinitely more likely than $D$ and $D$ infinitely more likely than $E$, that is, put $C$ before $D$ and $D$ before $E$.

Example 1,2 (Iterated admissibility) Consider $u_{2}$ illustrated in Table 2 which is obtained from the one in Example 11 by changing $u_{2}(B, E)$ from 1 to 4 . Here, $C$ could be optimal for player 2 if she believes that player 1 will use choice $A$, and $E$ could be optimal if she believes player 1 would use choice $B$, while $D$ could never be optimal whatever player 2 believes about player 1's choices. Hence, a lexicographic belief of player 1 assuming player 2's rationality should deem $C$ and $E$ infinitely more likely than $D$.

One motivation for developing lexicographic belief is to alleviate the tension between caution and rationality (Blume et al., 1991a, Brandenburger, 1992, Börgers, 1994, Samuelson, 1992, Börgers and Samuelson, 1994). All the three concepts tried to solve the tension by sacrificing rationality in different ways. Indeed, permissibility requires that the first level belief contains only rational choices, proper rationalizability requires that choices should be ordered according to the level of rationality, and iterated admissibility requires that choices which could be rational to be put in front of those which can never be. However, due to caution all allow occurrences of irrational choices. This sacrifice of rationality brought conceptual inconvenience since rationality is a basic assumption in game theory and is reasonable to be adopted as a criterion for each player's belief.

There is an approach which solves the tension without sacrificing rationality: using an incomplete information framework. That is, instead of considering the uncertainty about the opponents' rationality within a complete information framework, we take the uncertainty about the opponents' utility functions and consider types within the incomplete information framework. Then the occurrence of a irrational choice can be explained as that the "real" utility function of an opponent is different from the original one. Permissibility, proper rationalizability, and iterated admissibility can all be characterized within an incomplete information framework. This is the basic idea of this paper.

We use the above examples to explain this idea. 
Table 3 Alternative utility functions for player 2

\begin{tabular}{cccc}
\hline$v_{2}$ & $C$ & $D$ & $E$ \\
\hline$A$ & 2 & 3 & 1 \\
$B$ & 2 & 3 & 1
\end{tabular}

\begin{tabular}{cccc}
\hline$v_{2}^{\prime}$ & $C$ & $D$ & $E$ \\
\hline$A$ & 2 & 1 & 3 \\
$B$ & 2 & 1 & 3
\end{tabular}

Table 4 An alternative utility function for player 2

\begin{tabular}{llll}
\hline$w_{2}$ & $C$ & $D$ & $E$ \\
\hline$A$ & 3 & 5 & 4 \\
$B$ & 3 & 5 & 1 \\
\hline
\end{tabular}

Example 1,1 (Continued) As mentioned before, though only choice $C$ is rational for player 2, caution requires $D$ and $E$ to occur in player 1's belief as well. In a complete information framework, the occurrences of $D$ and $E$ are explained by player 2 's irrationality (i.e., "trembling hand"). In contrast, within an incomplete information framework they are explained by the possibility that the "real" utility function of player 2 is not $u_{2}$ but $v_{2}$ or $v_{2}^{\prime}$ in Table 3. Choice $D$ is optimal in $v_{2}$ and $E$ is optimal in $v_{2}^{\prime}$. In this manner, uncertainty about the opponent's rationality within a complete information framework is transformed into uncertainty about the opponent's real utility function within an incomplete information framework. It can be seen that primary belief in the opponent's rationality in complete information framework is equivalent to the condition that one deems $u_{2}$ or a utility function "very similar" to $u_{2}$ infinitely more likely to be the real utility function of player 2 than $v_{2}$ and $v_{2}^{\prime}$, and respecting the opponent's preferences is equivalent to the condition that those alternative utility functions should be ordered by their "similarity" to $u_{2}$.

Example1,2 (Continued) Since both $C$ and $E$ can be optimal in the original game, we only need a game to support choice $D$. Consider that the "real" utility function for player 2 is $w_{2}$ illustrated in Table 4 . Choice $D$ is optimal in $w_{2}$. It can be seen that assumption of player 2's rationality corresponds to that those utility function different from the original one to be less likely to the original one.

In this paper, we study the equivalences between conditions in complete and incomplete information models for 2-person static games and provide characterizations of permissibility, proper rationalizability, and assumption of rationality. First, we define the lexicographic epistemic model of a game with incomplete information. Then we give our characterization results which are separated into two groups. In the first group, we characterize permissibility and proper rationalizability, showing that a choice is permissible (properly rationalizable) within a complete information framework if and only if it is optimal for a belief hierarchy within the corresponding incomplete information framework that expresses common full belief in caution, primary belief in the opponent's utilities nearest to the original utilities (the opponent's utilities are centered around the original utilities), and a best (better) choice is supported by utilities nearest (nearer) to the original ones.

In the second group, we characterize permissibility in an alternative way and iterated admissibility, showing that a choice is permissible (iteratively admissible) 
within a complete information model if and only there is a belief hierarchy within the corresponding incomplete information framework that expresses common full belief of caution, rationality, and primary belief in the original utilities (prior assumption of the original utilities and every good choice is supported). We will further show in Section 5 5 that caution can be weakened in this group of characterizations.

Both proper rationalizability and iterated admissibility are refinements of permissibility (Perea, 2012). This can also be seen in the characterizations within the incomplete information models: in each group, each conditions for the latter is stronger than its counterpart for the former. The intrinsic difference between the two groups are the role of rationality: the first group does not need it, while the second group does. Nevertheless, we can construct a belief hierarchy which satisfies rationality and conditions in the first group. This corresponds to the fact that, within an complete information framework, it is always possible to have a belief hierarchy satisfying the conditions of proper rationalizability as well as those of iterated admissibility.

This paper is not the first one characterizing concepts in epistemic game theory within the incomplete information framework. Perea and Roy (2017) characterized $\varepsilon$-proper rationalizability in this approach by using a standard epistemic model without lexicographic beliefs. They showed that a type in a standard epistemic model with complete information expresses common full belief in caution and $\varepsilon$-trembling condition if and only if there is a type in the corresponding model with incomplete information sharing the same belief hierarchy with it which expresses common belief in caution, $\varepsilon$-centered belief around the original utilities $u$, and belief in rationality under the closest utility function. Since each properly rationalizable choice is the limit of a sequence of $\varepsilon$-proper rationalizable ones, the conditions adopted in their characterizations are very useful for us. Two conditions in our characterization of proper rationalizability, that is, caution and $u$-centered belief, are faithful translations of their conditions into lexicographic model. However, the most critical condition in their characterization, that is, belief in rationality under the closest utility function, is impossible to be adopted here. The reason is, as will be shown in Section 3 1, that a nearest utility function making a choice optimal does not always exist in lexicographic models. This is a salient difference between standard probabilistic beliefs and lexicographic ones. We define a weaker condition called "a better choice is supported by utilities nearer to the original one" and show that it can be used to characterize proper rationalizability.

Another essential difference between Perea and Roy (2017) and this paper is in the way of proof. Equivalence of belief hierarchies generated by types in models with complete and incomplete informations and type morphisms (Böge and Eisele, 1979, Heifetz and Samet, 1998, Perea and Kets, 2016) play important roles in Perea and Roy (2017)'s proof. In contrast, our proofs are based on constructing a specific correspondence between the two models. We show that conditions in a type of one model implies that appropriate conditions are satisfied in the corresponding type in the constructed model. Equivalence of hierarchies follows directly by construction. Our construction can also be used to prove Perea and Roy (2017)'s Theorem 6.1. Further, as will be discussed in Section [5,3, our construction shows that rationality is separable from other conditions in characterizing proper rationalizability. This con- 
firms that the consistency of caution and rationality within an incomplete information framework.

Our results, as well as Perea and Roy (2017)'s, also provide insights in decision theory and general epistemology. They imply that any choice permissible, properly rationalizable, or iteratively admissible within a complete information framework is also optimal for a belief satisfying corresponding conditions within an incomplete information framework, and vice versa. In other words, by just looking at the outcome, it is impossible to know the accurate epistemic situation behind the choice, that is, whether it is because of players' uncertainty about the opponents' rationality or uncertainty about what are the real utilities of the opponents.

The rest of the paper is organized as follows. Section 2 defines permissibility, proper rationalizability, and iterated admissibility in epistemic models with complete information. Section 3 introduces the lexicographic epistemic model with incomplete information and defines two groups of conditions on the types in such a model. Section 4 gives two groups of characterizations and their proofs. Section 5 gives some concluding remarks. Section 6 contains the proofs of all lemmas.

\section{Epistemic Model with Complete Information}

In this section, we give a survey of lexicographic epistemic model with complete information. Definitions here follow Perea (2012), Chapters 5-7.

Consider a finite 2-person static game $\Gamma=\left(C_{i}, u_{i}\right)_{i \in I}$ where $I=\{1,2\}$ is the set of players, $C_{i}$ is the finite set of choices and $u_{i}: C_{1} \times C_{2} \rightarrow \mathbb{R}$ is the utility function for player $i \in I$. In the following sometimes we denote $C_{1} \times C_{2}$ by $C$. We assume that each player has a lexicographic belief on the opponent's choices, a lexicographic belief on the opponent's lexicographic belief on her, and so on. This belief hierarchy is described by a lexicographic epistemic model with types.

Definition 21 (Epistemic model with complete information). Consider a finite 2person static game $\Gamma=\left(C_{i}, u_{i}\right)_{i \in I}$. A finite lexicographic epistemic model for $\Gamma$ is a tuple $M^{c o}=\left(T_{i}, b_{i}\right)_{i \in I}$ where

(a) $T_{i}$ is a finite set of types, and

(b) $b_{i}$ is a mapping that assigns to each $t_{i} \in T_{i}$ a lexicographic belief over $\Delta\left(C_{j} \times T_{j}\right)$, i.e., $b_{i}\left(t_{i}\right)=\left(b_{i 1}, b_{i 2}, \ldots, b_{i K}\right)$ where $b_{i k} \in \Delta\left(C_{j} \times T_{j}\right)$ for $k=1, \ldots, K$.

Consider $t_{i} \in T_{i}$ with $b_{i}\left(t_{i}\right)=\left(b_{i 1}, b_{i 2}, \ldots, b_{i K}\right)$. Each $b_{i k}(k=1, \ldots, K)$ is called $t_{i}$ 's level-k belief. For each $\left(c_{j}, t_{j}\right) \in C_{j} \times T_{j}$, we say $t_{i}$ deems $\left(c_{j}, t_{j}\right)$ possible iff $b_{i k}\left(c_{j}, t_{j}\right)>0$ for some $k \in\{1, \ldots, K\}$. We say $t_{i}$ deems $t_{j} \in T_{j}$ possible iff $t_{i}$ deems $\left(c_{j}, t_{j}\right)$ possible for some $c_{j} \in C_{j}$. For each $t_{i} \in T_{i}$, we denote by $T_{j}\left(t_{i}\right)$ the set of types in $T_{j}$ deemed possible by $t_{i}$.

A type $t_{i} \in T_{i}$ is cautious iff for each $c_{j} \in C_{j}$ and each $t_{j} \in T_{j}\left(t_{i}\right), t_{i}$ deems $\left(c_{j}, t_{j}\right)$ possible. That is, $t_{i}$ takes into account each choice of player $j$ for every belief hierarchy of $j$ deemed possible by $t_{i}$.

For each $c_{i} \in C_{i}$, let $u_{i}\left(c_{i}, t_{i}\right)=\left(u_{i}\left(c_{i}, b_{i 1}\right), \ldots, u_{i}\left(c_{i}, b_{i K}\right)\right)$ where for each $k=$ $1, \ldots, K, u_{i}\left(c_{i}, b_{i k}\right):=\Sigma_{\left(c_{j}, t_{j}\right) \in C_{j} \times T_{j}} b_{i k}\left(c_{j}, t_{j}\right) u_{i}\left(c_{i}, c_{j}\right)$, that is, each $u_{i}\left(c_{i}, b_{i k}\right)$ is the expected utility for $c_{i}$ over $b_{i k}$ and $u_{i}\left(c_{i}, t_{i}\right)$ is a vector of expected utilities. For each 
$c_{i}, c_{i}^{\prime} \in C_{i}$, we say that $t_{i}$ prefers $c_{i}$ to $c_{i}^{\prime}$, denoted by $u_{i}\left(c_{i}, t_{i}\right)>u_{i}\left(c_{i}^{\prime}, t_{i}\right)$, iff there is $k \in\{0, \ldots, K-1\}$ such that the following two conditions are satisfied:

(a) $u_{i}\left(c_{i}, b_{i \ell}\right)=u_{i}\left(c_{i}^{\prime}, b_{i \ell}\right)$ for $\ell=0, \ldots, k$, and

(b) $u_{i}\left(c_{i}, b_{i, k+1}\right)>u_{i}\left(c_{i}^{\prime}, b_{i, k+1}\right)$.

We say that $t_{i}$ is indifferent between $c_{i}$ and $c_{i}^{\prime}$, denoted by $u_{i}\left(c_{i}, t_{i}\right)=u_{i}\left(c_{i}^{\prime}, t_{i}\right)$, iff $u_{i}\left(c_{i}, b_{i k}\right)=u_{i}\left(c_{i}^{\prime}, b_{i k}\right)$ for each $k=1, \ldots, K$. It can be seen that this preference relation on $C_{i}$ under each type $t_{i}$ is a linear order. $c_{i}$ is rational (or optimal) for $t_{i}$ iff $t_{i}$ does not prefer any choice to $c_{i}$. A type $t_{i} \in T_{i}$ primarily believes in the opponent's rationality iff $t_{i}$ 's level-1 belief only assigns positive probability to those $\left(c_{j}, t_{j}\right)$ where $c_{j}$ is rational for $t_{j}$. That is, at least in the primary belief $t_{i}$ is convinced that $j$ behaves rationally given her belief.

For $\left(c_{j}, t_{j}\right),\left(c_{j}^{\prime}, t_{j}^{\prime}\right) \in C_{j} \times T_{j}$, we say that $t_{i}$ deems $\left(c_{j}, t_{j}\right)$ infinitely more likely than $\left(c_{j}^{\prime}, t_{j}^{\prime}\right)$ iff there is $k \in\{0, \ldots, K-1\}$ such that the following two conditions are satisfied:

(a) $b_{i \ell}\left(c_{j}, t_{j}\right)=b_{i \ell}\left(c_{j}^{\prime}, t_{j}^{\prime}\right)=0$ for $\ell=0, \ldots, k$, and

(b) $b_{i, k+1}\left(c_{j}, t_{j}\right)>0$ and $b_{i, k+1}\left(c_{j}^{\prime}, t_{j}^{\prime}\right)=0$.

A cautious type $t_{i} \in T_{i}$ respects the opponent's preferences iff for each $t_{j} \in T_{j}\left(t_{i}\right)$ and $c_{j}, c_{j}^{\prime} \in C_{j}$ where $t_{j}$ prefers $c_{j}$ to $c_{j}^{\prime}, t_{i}$ deems $\left(c_{j}, t_{j}\right)$ infinitely more likely than $\left(c_{j}^{\prime}, t_{j}\right)$. That is, $t_{i}$ arranges $j$ 's choices from the most to the least preferred for each belief hierarchy of $j$ deemed possible by $t_{i}$. It can be seen that respect of the opponent's preferences implies primary belief in the opponent's rationality. Indeed, the former requires that each type of the opponent deemed possible in the primary belief should only pair with choices most preferred under that type.

Let $P$ be an arbitrary property of lexicographic beliefs. We define that (a) $t_{i} \in T_{i}$ expresses 0 -fold full belief in $P$ iff $t_{i}$ satisfies $P$;

(b) For each $n \in \mathbb{N}, t_{i} \in T_{i}$ expresses $(n+1)$-fold full belief in $P$ iff $t_{i}$ only deems possible $j$ 's types that express $n$-fold full belief in $P$.

A type $t_{i}$ expresses common full belief in $P$ iff it expresses $n$-fold full belief in $P$ for each $n \in \mathbb{N}$.

Definition 2,2 (Permissibility and proper rationalizability). Consider a finite lexicographic epistemic model $M^{c o}=\left(T_{i}, b_{i}\right)_{i \in I}$ for a game $\Gamma=\left(C_{i}, u_{i}\right)_{i \in I} . c_{i} \in C_{i}$ is permissible iff it is rational for some $t_{i} \in T_{i}$ which expresses common full belief in caution and primary belief in rationality. $c_{i}$ is properly rationalizable iff it is rational for some $t_{i} \in T_{i}$ which expresses common full belief in caution and respect of preferences.

Since respect of the opponent's preferences implies primary belief in the opponent's rationality, proper rationalizability implies permissibility, while the reverse does not hold.

A cautious type $t_{i} \in T_{i}$ assumes the j's rationality iff the following two conditions are satisfied:

(a) for all of player $j$ 's choices $c_{j}$ that are optimal for some cautious belief, $t_{i}$ deems possible some cautious type $t_{j}$ for which $c_{j}$ is optimal; 
(b) $t_{i}$ deems all choice-type pairs $\left(c_{j}, t_{j}\right)$ where $t_{j}$ is cautious and $c_{j}$ is optimal for $t_{j}$ infinitely more likely than any choice-type pairs $\left(c_{j}^{\prime}, t_{j}^{\prime}\right)$ that does not have this property.

Informally speaking, assumption of the opponent's rationality is that $t_{i}$ puts all "good" choices in front of those "bad" choices.

On the other hand, extending assumption of rationality into $n$-fold for any $n \in \mathbb{N}$ is more complicated than $n$-fold full belief. Formally, consider a finite lexicographic epistemic model $M^{c o}=\left(T_{i}, b_{i}\right)_{i \in I}$ for a game $\Gamma=\left(C_{i}, u_{i}\right)_{i \in I}$. A cautious type $t_{i} \in T_{i}$ expresses 1-fold assumption of rationality iff it assumes $j$ 's rationality. For any $n \in \mathbb{N}$, we say that a cautious type $t_{i} \in T_{i}$ expresses $(n+1)$-fold assumption of rationality iff the following two conditions are satisfied:

(a) whenever a choice $c_{j}$ of player $j$ is optimal for some cautious type (not necessarily in $M^{c o}$ ) that expresses up to $n$-fold assumption of rationality, $t_{i}$ deems possible some cautious type $t_{j}$ for player $j$ which expresses up to $n$-fold assumption of rationality for which $c_{j}$ is optimal;

(b) $t_{i}$ deems all choice-type pair $\left(c_{j}, t_{j}\right)$ where $t_{j}$ is cautious and expresses up to $n$ fold assumption of rationality and $c_{j}$ is optimal for $t_{j}$ infinitely more likely than any choice-type pair $\left(c_{j}^{\prime}, t_{j}^{\prime}\right)$ that does not satisfy this property.

We say that $t_{i}$ expresses common assumption of rationality iff it expresses $n$-fold assumption of rationality for every $n \in \mathbb{N}$.

Definition 2,3 (Iterated admissibility). Consider a finite lexicographic epistemic model $M^{c o}=\left(T_{i}, b_{i}\right)_{i \in I}$ for a game $\Gamma=\left(C_{i}, u_{i}\right)_{i \in I} . c_{i}$ is iteratively admissible iff it is rational for some $t_{i} \in T_{i}$ which expresses common assumption of rationality.

\section{Epistemic Model with Incomplete Information}

In this section, we define the lexicographic epistemic model with incomplete information which is the counterpart of the probabilistic epistemic model with incomplete information introduced by Battigalli (2003) and extensively developed in Battigalli and Siniscalchi (2003), (2007), and Dekel and Siniscalchi (2015). We also define conditions on types in such a model.

Definition 3,1 (Lexicographic epistemic model with incomplete information). Consider a finite 2-person static game form $G=\left(C_{i}\right)_{i \in I}$. For each $i \in I$, let $V_{i}$ be the set of utility functions $v_{i}: C_{1} \times C_{2} \rightarrow \mathbb{R}$. A finite lexicographic epistemic model for $G$ with incomplete information is a tuple $M^{i n}=\left(\Theta_{i}, w_{i}, \beta_{i}\right)_{i \in I}$ where

(a) $\Theta_{i}$ is a finite set of types,

(b) $w_{i}$ is a mapping that assigns to each $\theta_{i} \in \Theta_{i}$ a utility function $w_{i}\left(\theta_{i}\right) \in V_{i}$, and

(c) $\beta_{i}$ is a mapping that assigns to each $\theta_{i} \in \Theta_{i}$ a lexicographic belief over $\Delta\left(C_{j} \times \Theta_{j}\right)$, i.e., $\beta_{i}\left(\theta_{i}\right)=\left(\beta_{i 1}, \beta_{i 2}, \ldots, \beta_{i K}\right)$ where $\beta_{i k} \in \Delta\left(C_{j} \times \Theta_{j}\right)$ for $k=1, \ldots, K$.

Concepts such as " $\theta_{i}$ deems $\left(c_{j}, \theta_{j}\right)$ possible" and " $\theta_{i}$ deems $\left(c_{j}, \theta_{j}\right)$ infinitely more likely than $\left(c_{j}^{\prime}, \theta_{j}^{\prime}\right)$ " can be defined in a similar way as in Section 2 For each $\theta_{i} \in \Theta_{i}$, we use $\Theta_{j}\left(\theta_{i}\right)$ to denote the set of types in $\Theta_{j}$ deemed possible by $\theta_{i}$. For 
each $\theta_{i} \in \Theta_{i}$ and $v_{i} \in V_{i}, \theta_{i}^{v_{i}}$ is the auxiliary type satisfying that $\beta_{i}\left(\theta_{i}^{v_{i}}\right)=\beta_{i}\left(\theta_{i}\right)$ and $w_{i}\left(\theta_{i}^{v_{i}}\right)=v_{i}$.

For each $c_{i} \in C_{i}, v_{i} \in V_{i}$, and $\theta_{i} \in \Theta_{i}$ with $\beta_{i}\left(\theta_{i}\right)=\left(\beta_{i 1}, \beta_{i 2}, \ldots, \beta_{i K}\right)$, let $v_{i}\left(c_{i}, \theta_{i}\right)=$ $\left(v_{i}\left(c_{i}, \beta_{i 1}\right), \ldots, v_{i}\left(c_{i}, \beta_{i K}\right)\right)$ where $v_{i}\left(c_{i}, \beta_{i k}\right):=\Sigma_{\left(c_{j}, \theta_{j}\right) \in C_{j} \times \Theta_{j}} \beta_{i k}\left(c_{j}, \theta_{j}\right) v_{i}\left(c_{i}, c_{j}\right)$ for each $k=1, \ldots, K$, For each $c_{i}, c_{i}^{\prime} \in C_{i}$ and $\theta_{i} \in \Theta_{i}$, we say that $\theta_{i}$ prefers $c_{i}$ to $c_{i}^{\prime}$ iff $w_{i}\left(\theta_{i}\right)\left(c_{i}, \theta_{i}\right)$ $>w_{i}\left(\theta_{i}\right)\left(c_{i}^{\prime}, \theta_{i}\right)$. As in Section 2, this is also the lexicographic comparison between two vectors. $c_{i}$ is rational (or optimal) for $\theta_{i}$ iff $\theta_{i}$ does not prefer any choice to $c_{i}$.

In the following we define two groups of conditions on types in an epistemic model with incomplete information, which correspond to the two groups of characterizations in Section 4

\subsection{The first group of conditions}

Definition 32 (Caution). A type $\theta_{i} \in \Theta_{i}$ is cautious iff for each $c_{j} \in C_{j}$ and each $\theta_{j} \in$ $\Theta_{j}\left(\theta_{i}\right)$, there is some utility function $v_{j} \in V_{j}$ such that $\theta_{i}$ deems $\left(c_{j}, \theta_{j}^{v_{j}}\right)$ possible.

This is a faithful translation of Perea and Roy (2017)'s definition of caution in a probabilistic model into a lexicographic one. It is the counterpart of caution defined within the complete information framework in Section 2, the only difference is that in incomplete information models we allow different utility functions since $c_{j}$ will be required to be rational for the paired type.

For each $u_{i}, v_{i} \in V_{i}$, we define the distance $d\left(u_{i}, v_{i}\right)$ between $u_{i}, v_{i}$ by $d\left(u_{i}, v_{i}\right)=$ $\left[\Sigma_{s \in S}\left(u_{i}(s)-v_{i}(s)\right)^{2}\right]^{1 / 2}$. This is the Euclidean distance on $\mathbb{R}^{C}$. We choose it is just out of simplicity. Any distance satisfying the three conditions in Section 3.3 of Perea and Roy (2017) also works in our characterization.

A problem here is that utility functions are numerical representations of preferences, yet the Euclidean distance measures cardinal similarity between utility functions rather than the similarity between preferences they represent. For example, though multiplying $u_{i}$ with a positive number leads to the same preferences represented by $u_{i}$, its Euclidean distance from $u_{i}$ may be large. In Section 54 we will define an ordinal distance on $V_{i}$ and show that the characterizations still hold under that distance.

Definition 3, 3 (Primary belief in utilities nearest to $u$ and $u$-centered belief). Consider a static game form $G=\left(C_{i}\right)_{i \in I}$, a lexicographic epistemic model $M^{i n}=$ $\left(\Theta_{i}, w_{i}, \beta_{i}\right)_{i \in I}$ for $G$ with incomplete information, and a pair $u=\left(u_{i}\right)_{i \in I}$ of utility functions.

(3.1) A type $\theta_{i} \in \Theta_{i}$ primarily believes in utilities nearest to $u$ iff $\theta_{i}$ 's level-1 belief only assigns positive probability to $\left(c_{j}, \theta_{j}\right)$ which satisfies that $d\left(w_{j}\left(\theta_{j}\right), u_{j}\right) \leq$ $d\left(w_{j}\left(\theta_{j}^{\prime}\right), u_{j}\right)$ for all $\theta_{j}^{\prime} \in \Theta_{j}\left(\theta_{i}\right)$ with $\beta_{j}\left(\theta_{j}^{\prime}\right)=\beta_{j}\left(\theta_{j}\right)$.

(3.2) A type $\theta_{i} \in \Theta_{i}$ has $u$-centered belief iff for any $c_{j}, c_{j}^{\prime} \in C_{j}$, any $\theta_{j} \in \Theta_{j}$, and any $v_{j}, v_{j}^{\prime} \in V_{j}$ such that $\left(c_{j}, \theta_{j}^{v_{j}}\right)$ and $\left(c_{j}^{\prime}, \theta_{j}^{v_{j}^{\prime}}\right)$ are deemed possible by $\theta_{i}$, it holds that $\theta_{i}$ deems $\left(c_{j}, \theta_{j}^{v_{j}}\right)$ infinitely more likely than $\left(c_{j}^{\prime}, \theta_{j}^{v_{j}^{\prime}}\right)$ whenever $d\left(v_{j}, u_{j}\right)<d\left(v_{j}^{\prime}, u_{j}\right)$.

Definition 3,3 gives restrictions on the order of types in a lexicographic belief. (3.1) requires that $\theta_{i}$ primarily believes in type $\theta_{j}$ only if $\theta_{j}$ 's utility function is the 
Table 5 No nearest utility function in lexicographic beliefs

\begin{tabular}{cccc}
\hline$u_{1}$ & $D$ & $E$ & $F$ \\
\hline$A$ & 1 & 1 & 1 \\
$B$ & 1 & 1 & 0 \\
$C$ & 1 & 0 & 1 \\
\hline
\end{tabular}

nearest one to $u_{j}$ among all types deemed possible by $\theta_{i}$ which share the same belief with $\theta_{j}$. (3.2) requires that the types of $j$ sharing the same belief deemed possible by $\theta_{i}$ are arranged according to the distance of their assigned utility functions from $u_{j}$ : the farther a type $\theta_{j}$ 's utility function is from $u_{j}$, the later $\theta_{j}$ occurs in the lexicographic belief of $\theta_{i}$. (3.2) is a faithful translation of Perea and Roy (2017)'s Definition 3.2 into lexicographic model and (3.1) is weaker than (3.2).

The essential difference between our conditions and Perea and Roy (2017)'s lies in the following definition.

Definition 34 (A best (better) choice is supported by utilities nearest (nearer) to $u$ ). Consider a static game form $G=\left(C_{i}\right)_{i \in I}$, a lexicographic epistemic model $M^{i n}=\left(\Theta_{i}, w_{i}, \beta_{i}\right)_{i \in I}$ for $G$ with incomplete information, and a pair $u=\left(u_{i}\right)_{i \in I}$ of utility functions.

(4.1) A type $\theta_{i} \in \Theta_{i}$ believes in that a best choice of $j$ is supported by utilities nearest to $u$ iff for any $\left(c_{j}, \theta_{j}\right),\left(c_{j}^{\prime}, \theta_{j}^{\prime}\right)$ deemed possible by $\theta_{i}$ with $\beta_{j}\left(\theta_{j}\right)=\beta_{j}\left(\theta_{j}^{\prime}\right)$, if $c_{j}$ is optimal for $\beta_{j}\left(\theta_{j}\right)$ in $u_{j}$ but $c_{j}^{\prime}$ is not, then $d\left(w_{j}\left(\theta_{j}\right), u_{j}\right)<d\left(w_{j}\left(\theta_{j}^{\prime}\right), u_{j}\right)$.

(4.2) A type $\theta_{i} \in \Theta_{i}$ believes in that a better choice of $j$ is supported by utilities nearer to $u$ iff for any $\left(c_{j}, \theta_{j}\right),\left(c_{j}^{\prime}, \theta_{j}^{\prime}\right)$ deemed possible by $\theta_{i}$ with $\beta_{j}\left(\theta_{j}\right)=\beta_{j}\left(\theta_{j}^{\prime}\right)$, if $u_{j}\left(c_{j}, \theta_{j}\right)>u_{j}\left(c_{j}^{\prime}, \theta_{j}^{\prime}\right)$, then $d\left(w_{j}\left(\theta_{j}\right), u_{j}\right)<d\left(w_{j}\left(\theta_{j}^{\prime}\right), u_{j}\right)$.

Definition 34 gives restriction on the relation between paired choices and types. (4.1) requires that for each belief of player $j$, a choice optimal for that belief should be supported by the nearest utility function to $u_{j}$. (4.2) requires that for each belief of player $j$, a utility function supporting a "better" choice (i.e., $c_{j}$ ) should be nearer to $u_{j}$ than one supporting a "worse" choice (i.e., $c_{j}^{\prime}$ ). It can be seen that (4.2) is stronger than (4.1).

(4.2) is similar to Perea and Roy (2017)'s Definition 3.3 which requires that for each $\left(c_{j}, \theta_{j}\right)$ deemed possible by $\theta_{i}, w_{j}\left(\theta_{j}\right)$ is the nearest utility function in $V_{j}$ to $u_{j}$ among those in which $c_{j}$ is rational under $\beta_{j}\left(\theta_{j}\right)$. It can be shown by Lemmas 5.4 and 5.5 in Perea and Roy (2017) that Definition 34 (4.2) is weaker than Perea and Roy [21]'s Definition 3.3. We adopt it here since a nearest utility function does not in general exist for lexicographic beliefs. That is, given $u_{j} \in V_{j}, c_{j} \in C_{j}$, and a lexicographic belief $\beta_{j}$, there may not exist $v_{j} \in V_{j}$ satisfying that (1) $c_{j}$ is rational at $v_{j}$ under $\beta_{j}$, and (2) there is no $v_{j}^{\prime} \in V_{j}$ such that $c_{j}$ is rational at $v_{j}^{\prime}$ for $\beta_{j}$ and $d\left(v_{j}^{\prime}, u_{j}\right)<d\left(v_{j}, u_{j}\right)$. See the following example.

Example 3,1 (No nearest utility function). Consider a game $\Gamma$ where player 1 has choices $A, B$, and $C$ and player 2 has choices $D, E$, and $F$. The payoff function $u_{1}$ of player 1 is shown in Table 5. Let $\beta_{1}=(D, E, F)$, that is, player 1 deems player 2's 
Table 6 A worse choice is supported by a better utility function

\begin{tabular}{llll}
\hline$u_{1}$ & $D$ & $E$ & $F$ \\
\hline$A$ & 1 & 1 & 1 \\
$B$ & $1+\frac{d}{2}$ & 1 & 0 \\
$C$ & 1 & 0 & 1 \\
\hline
\end{tabular}

choice $D$ infinitely more likely than $E$ and $E$ infinitely more likely than $F$. In $u_{1}, A$ is rational for $\beta_{1}$ but $B$ is not.

Now we show that there is no nearest utility function to $u_{1}$ at which $B$ is rational under $\beta_{1}$. Suppose there is such a function $v_{1} \in V_{1}$. Let $d=d\left(v_{1}, u_{1}\right)$. It can be seen that $d>0$. Consider $v_{1}^{\prime}$ in Table $6 . B$ is also rational at $v_{1}^{\prime}$ under $\beta_{1}$, while $d\left(v_{1}^{\prime}, u_{1}\right)=$ $\frac{d}{2}<d=d\left(v_{1}, u_{1}\right)$, a contradiction. Also, though $\beta_{1}$ prefers $B$ to $C$ in $u_{1}$, it can be seen that for each utility function $v_{1}^{B}$ in which $B$ is rational under $\beta_{1}$, there is some $v_{1}^{C} \in V_{1}$ satisfying (1) $C$ is optimal in $v_{1}^{C}$ under $\beta_{1}$, and (2) $d\left(v_{1}^{C}, u_{1}\right)<d\left(v_{1}^{B}, u_{1}\right)$. Indeed, this can be done by letting $v_{1}^{C}(C, D)=1+d\left(v_{1}^{B}, u_{1}\right) / 2$ and $v_{1}^{C}\left(c_{1}, c_{2}\right)=u_{1}\left(c_{1}, c_{2}\right)$ for all other $\left(c_{1}, c_{2}\right) \in C_{1} \times C_{2}$.

Example 3 , 1 shows that the relationship between preferences among choices and the distance of utility functions from the original one is more complicated for lexicographic beliefs. That is why we adopt Definition 344 (4.2) here. The following lemma guarantees the existence of utility functions satisfying the condition in Definition 34 (4.2). It shows that, given a utility function $u_{i}$ and a lexicographic belief $\beta_{i}$, corresponding to the sequence $c_{i 1}, \ldots, c_{i N}$ of $i$ 's choices arranged from the most to the least preferred at $u_{i}$ under $\beta_{i}$, there is a sequence $v_{i 1}, \ldots, v_{i N}$ of utility functions arranged from the nearest to the farthest to $u_{i}$ such that for each $n=1, \ldots, N, c_{i n}$ is rational at $v_{i n}$ under $\beta_{i}$. This lemma plays a similar role in our characterizations as Lemmas 5.4 and 5.5 in Perea and Roy [21].

Lemma 3,1 (Existence of utilities satisfying Definition 3,4 (4.2)). Consider a static game form $G=\left(C_{i}\right)_{i \in I}, u_{i} \in V_{i}$, and $\beta_{i}=\left(\beta_{i 1}, \beta_{i 2}, \ldots, \beta_{i K}\right)$ such that $\beta_{i k} \in \Delta\left(C_{j}\right)$ for each $k=1, \ldots, K$. Let $\Pi_{i}\left(\beta_{i}\right)=\left(C_{i 1}, C_{i 2}, \ldots, C_{i L}\right)$ be a partition of $C_{i}$ satisfying that (1) for each $\ell=1, \ldots, L$ and each $c_{i \ell}, c_{i \ell}^{\prime} \in C_{i \ell}, u_{i}\left(c_{i \ell}, \beta_{i}\right)=u_{i}\left(c_{i \ell}^{\prime}, \beta_{i}\right)$, and (2) for each $\ell=1, \ldots, L-1$, each $c_{i \ell} \in C_{i \ell}$ and $c_{i, \ell+1} \in C_{i, \ell+1}, u_{i}\left(c_{i \ell}, \beta_{i}\right)>u_{i}\left(c_{i, \ell+1}, \beta_{i}\right)$. That is, $\Pi_{i}\left(\beta_{i}\right)$ is the sequence of equivalence classes of choices in $C_{i}$ arranged from the most preferred to the least preferred under $\beta_{i}$.

Then there are $v_{i 1}, \ldots, v_{i L} \in V_{i}$ satisfying

(a) $v_{i 1}=u_{i}$,

(b) For each $\ell=1, \ldots, L$ and each $c_{i \ell} \in C_{i \ell}, c_{i \ell}$ is rational at $v_{i \ell}$ under $\beta_{i}$, and

(c) For each $\ell=1, \ldots, L-1, d\left(v_{i \ell}, u_{i}\right)<d\left(v_{i, \ell+1}, u_{i}\right)$.

3.2 The second group of conditions

Definition 3,5 (Belief in rationality). $\theta_{i} \in \Theta_{i}$ believes in $j$ 's rationality iff $\theta_{i}$ deems $\left(c_{j}, \theta_{j}\right)$ possible only if $c_{j}$ is rational for $\theta_{j}$. 
In an incomplete information model, since each type is assigned with a belief on the opponent's choice-type pairs as well as a payoff function, caution and a full belief of rationality can be satisfied simultaneously. The consistency of caution and rationality is the essential difference of models with incomplete information from those with complete information. Rationality does not appear in the first group of characterizations, but in the proofs we will construct incomplete information models whose types satisfies all the conditions as well as common full belief in rationality. On the other hand, rationality plays an important role in the characterization of the second group. We will discuss more about this consistency between caution and rationality in Sections 53 .

Definition 3,6 (Primary belief in $u$ and prior assumption of $u$ ). Consider a static game form $G=\left(C_{i}\right)_{i \in I}$, a lexicographic epistemic model $M^{i n}=\left(\Theta_{i}, w_{i}, \beta_{i}\right)_{i \in I}$ for $G$ with incomplete information, and a pair $u=\left(u_{i}\right)_{i \in I}$ of utility functions.

(6.1) $\theta_{i} \in \Theta_{i}$ primarily believes in $u$ iff $\theta_{i}$ 's level-1 belief only assigns positive probability to $\left(c_{j}, \theta_{j}\right)$ with $w_{j}\left(\theta_{j}\right)=u_{j}$.

(6.2) $\theta_{i} \in \Theta_{i}$ prior assumes $u$ iff for any $\left(c_{j}, \theta_{j}\right)$ with $\theta_{j}$ cautious deemed possible by $\theta_{i}$ satisfying that $w_{j}\left(\theta_{j}\right)=u_{j}$, then $\theta_{i}$ deems $\left(c_{j}, \theta_{j}\right)$ infinitely more likely than any pair does not satisfy that property.

Primary belief in $u$ is stronger than Definition 33 (3.1). (3.1) allows the occurrence of a type with a utility function which is very similar (but not necessarily equal) to $u_{j}$ in the level-1 belief of $\theta_{i}$, while primary belief in $u$ only allows types with utility function $u_{j}$ there. (6.2) is stronger than (6.1) since (6.2) requires that all choice-type pairs which believes in $u$ should be put in front of pairs which do not believe in $u$, while (6.1) only requires that level-1 belief believes in $u$.

Definition 3 , (Every good choice is supported). Consider a static game form $G=$ $\left(C_{i}\right)_{i \in I}$, a lexicographic epistemic model $M^{i n}=\left(\Theta_{i}, w_{i}, \beta_{i}\right)_{i \in I}$ for $G$ with incomplete information, and a pair $u=\left(u_{i}\right)_{i \in I}$ of utility functions. A cautious type $\theta_{i} \in \Theta_{i}$ assumes that every good choice of $j$ is supported iff for each $c_{j}$ that is optimal for some cautious type of $j$ (may not be in $M^{i n}$ ) with $u_{j}$ as its assigned utility function, $\theta_{i}$ deems possible a cautious type $\theta_{j} \in \Theta_{j}$ such that $w_{j}\left(\theta_{j}\right)=u_{j}$ and $c_{j}$ is optimal for $\theta_{j}$.

Common assumption of prior $u$ and that every good choice is supported is, as common assumption of rationality in incomplete information model, more complicated from common full belief. We have the following definition.

Definition 3,8 ( $n$-fold assumption of prior $u$ and that every good choice is supported) Consider a static game form $G=\left(C_{i}\right)_{i \in I}$, a lexicographic epistemic model $M^{i n}=\left(\Theta_{i}, w_{i}, \beta_{i}\right)_{i \in I}$ for $G$ with incomplete information, and a pair $u=\left(u_{i}\right)_{i \in I}$ of utility functions. $\theta_{i} \in \Theta_{i}$ expresses 1-fold assumption of prior $u$ and that every good choice is supported iff it prior assumes $u$ and assumes that every good choice of $j$ is supported. For any $n \in \mathbb{N}$, we say that a cautious type $\theta_{i} \in \Theta_{i}$ expresses $(n+1)$-fold assumption of prior $u$ and that every good choice is supported iff the following two conditions are satisfied:

(a) whenever a choice $c_{j}$ of player $j$ is optimal for some cautious type (not necessarily in $M^{i n}$ ) with $u_{j}$ as its assigned utility function that expresses up to $n$-fold assumption 
of that every good choice is supported, $\theta_{i}$ deems possible some cautious type $\theta_{j}$ with $w_{j}\left(\theta_{j}\right)=u_{j}$ for player $j$ which expresses up to $n$-fold assumption of prior $u$ and that every good choice is supported for which $c_{j}$ is optimal.

(b) $\theta_{i}$ deems all choice-type pairs $\left(c_{j}, \theta_{j}\right)$, where $\theta_{j}$ is cautious and expresses up to $n$-fold assumption of prior $u$ and that every good choice is supported and satisfies $w_{j}\left(\theta_{j}\right)=u_{j}$, infinitely more likely than any choice-type pair $\left(c_{j}^{\prime}, \theta_{j}^{\prime}\right)$ that does not satisfy this property.

We say that $t_{i}$ expresses common assumption of prior $u$ and in that every good choice is supported iff it expresses $n$-fold assumption of prior $u$ and that every good choice is supported for every $n \in \mathbb{N}$.

\section{Characterizations}

So far we have introduced conditions under two different frameworks for static games: one includes permissibility, proper rationalizability, and iterated admissibility within a complete information framework, the other contains various conditions on types within an incomplete information framework. In this section we will show that there are correspondences between them. The characterizations will be separated into two groups: in the first group we characterize permissibility and proper rationalizability, and in the second group we characterize permissibility in an alternative way and iterated admissibility. The critical difference between the two groups is the role of rationality.

\subsection{Without rationality: permissibility and proper rationalizability}

In this subsection we give characterizations of permissibility and proper rationalizability. An illustrative example will also be provided.

Theorem 4,1 (Characterization of permissibility). Consider a finite 2-person static game $\Gamma=\left(C_{i}, u_{i}\right)_{i \in I}$ and the corresponding game form $G=\left(C_{i}\right)_{i \in I}$.

Then, $c_{i}^{*} \in C_{i}$ is permissible if and only if there is some finite lexicographic epistemic model $M^{i n}=\left(\Theta_{i}, w_{i}, \beta_{i}\right)_{i \in I}$ with incomplete information for $G$ and some $\theta_{i}^{*} \in \Theta_{i}$ with $w_{i}\left(\theta_{i}^{*}\right)=u_{i}$ such that

(a) $c_{i}^{*}$ is rational for $\theta_{i}^{*}$, and,

(b) $\theta_{i}^{*}$ expresses common full belief in caution, primary belief in utilities nearest to $u$, and that a best choice is supported by utilities nearest to $u$.

Theorem 4,2 (Characterization of proper rationalizability). Consider a finite 2person static game $\Gamma=\left(C_{i}, u_{i}\right)_{i \in I}$ and the corresponding game form $G=\left(C_{i}\right)_{i \in I}$.

Then, $c_{i}^{*} \in C_{i}$ is properly rationalizable if and only if there is some finite lexicographic epistemic model $M^{i n}=\left(\Theta_{i}, w_{i}, \beta_{i}\right)_{i \in I}$ for $G$ and some $\theta_{i}^{*} \in \Theta_{i}$ with $w_{i}\left(\theta_{i}^{*}\right)=$ $u_{i}$ such that

(a) $c_{i}^{*}$ is rational for $\theta_{i}^{*}$, and

(b) $\theta_{i}^{*}$ expresses common full belief in caution, $u$-centered belief, and that a better choice is supported by utilities nearer to $u$. 
Table 7 The game for Example 41

\begin{tabular}{llll}
\hline$u_{1} \backslash u_{2}$ & $D$ & $E$ & $F$ \\
\hline$A$ & 0,3 & 1,2 & 1,1 \\
$B$ & 1,3 & 0,2 & 1,1 \\
$C$ & 1,3 & 1,2 & 0,1 \\
\hline
\end{tabular}

Table 8 Alternative utility functions for players 1 and 2

\begin{tabular}{llll}
\hline$v_{1}$ & $D$ & $E$ & $F$ \\
\hline$A$ & 0 & 1 & 1 \\
$B$ & 2 & 0 & 1 \\
$C$ & 1 & 1 & 0 \\
\hline
\end{tabular}

\begin{tabular}{llll}
\hline$v_{1}^{\prime}$ & $D$ & $E$ & $F$ \\
\hline$A$ & 3 & 1 & 1 \\
$B$ & 2 & 0 & 1 \\
$C$ & 1 & 1 & 0 \\
\hline
\end{tabular}

\begin{tabular}{llll}
\hline$v_{2}$ & $D$ & $E$ & $F$ \\
\hline$A$ & 3 & 2 & 1 \\
$B$ & 3 & 2 & 1 \\
$C$ & 3 & 4 & 1 \\
\hline
\end{tabular}

\begin{tabular}{llll}
\hline$v_{2}^{\prime}$ & $D$ & $E$ & $F$ \\
\hline$A$ & 3 & 2 & 1 \\
$B$ & 3 & 2 & 1 \\
$C$ & 3 & 4 & 5 \\
\hline
\end{tabular}

To show these statements, we will construct a correspondence between complete information models and incomplete ones and show that conditions on a type in one model can be transformed into proper conditions on the corresponding type in the constructed model. We use the following example to show the intuition.

Example 4,1. Consider the game $\Gamma$ in Table 7(Perea, 2012, p.190) and the lexicographic model $M^{c o}=\left(T_{i}, b_{i}\right)_{i \in I}$ for $\Gamma$ where $T_{1}=\left\{t_{1}\right\}, T_{2}=\left\{t_{2}\right\}$, and

$$
b_{1}\left(t_{1}\right)=\left(\left(D, t_{2}\right),\left(E, t_{2}\right),\left(F, t_{2}\right)\right), b_{2}\left(t_{2}\right)=\left(\left(C, t_{1}\right),\left(B, t_{1}\right),\left(A, t_{1}\right)\right) .
$$

It can be seen that $D$ is properly rationalizable (and therefore permissible) since it is rational for $t_{2}$ which expresses common full belief in caution and respect of preferences. Consider the lexicographic epistemic model $M^{i n}=\left(\Theta_{i}, w_{i}, \beta_{i}\right)_{i \in I}$ with incomplete information for the corresponding game form where $\Theta_{1}=\left\{\theta_{11}, \theta_{12}, \theta_{13}\right\}$, $\Theta_{2}=\left\{\theta_{21}, \theta_{22}, \theta_{23}\right\}$, and

$$
\begin{aligned}
& w_{1}\left(\theta_{11}\right)=u_{1}, \beta_{1}\left(\theta_{11}\right)=\left(\left(D, \theta_{21}\right),\left(E, \theta_{22}\right),\left(F, \theta_{23}\right)\right), \\
& w_{1}\left(\theta_{12}\right)=v_{1}, \beta_{1}\left(\theta_{12}\right)=\left(\left(D, \theta_{21}\right),\left(E, \theta_{22}\right),\left(F, \theta_{23}\right)\right), \\
& w_{1}\left(\theta_{13}\right)=v_{1}^{\prime}, \beta_{1}\left(\theta_{13}\right)=\left(\left(D, \theta_{21}\right),\left(E, \theta_{22}\right),\left(F, \theta_{23}\right)\right), \\
& \left.w_{2}\left(\theta_{21}\right)=u_{2}, \beta_{2}\left(\theta_{21}\right)=\left(\left(C, \theta_{11}\right),\left(B, \theta_{12}\right)\right),\left(A, \theta_{13}\right)\right), \\
& \left.w_{2}\left(\theta_{22}\right)=v_{2}, \beta_{2}\left(\theta_{22}\right)=\left(\left(C, \theta_{11}\right),\left(B, \theta_{12}\right)\right),\left(A, \theta_{13}\right)\right), \\
& \left.w_{2}\left(\theta_{23}\right)=v_{2}^{\prime}, \beta_{2}\left(\theta_{23}\right)=\left(\left(C, \theta_{11}\right),\left(B, \theta_{12}\right)\right),\left(A, \theta_{13}\right)\right) .
\end{aligned}
$$

where the alternative utility functions are shown in Table 8 . For each $i \in I, \theta_{i 1}, \theta_{i 2}$, and $\theta_{i 3}$ have the same belief; the only difference lies in their assigned utility functions since each should support some choice. The relation between $M^{\text {in }}$ and $M^{c o}$ can be seen clearly: for each $i \in I, \theta_{i 1}, \theta_{i 2}$, and $\theta_{i 3}$ correspond to $t_{i}$ in the sense that the belief of the former is obtained by replacing every occurrence of $t_{j}$ in the belief of $t_{i}$ by the type corresponding to $t_{j}$ in $M^{i n}$ at which the paired choice is optimal. It can be seen that $\theta_{11}$ expresses common full belief in caution, $u$-centered belief, and that a better choice is supported by utilities nearer to $u$ (therefore primary belief in utilities nearest to $u$ and that a best choice is supported by utilities nearest to $u$ ). Also, since the assigned utility function of $\theta_{11}$ is $u_{1}, C$ is rational for $\theta_{11}$. 
4.2 With rationality: permissibility and iterated admissibility

In this subsection we give an alternative characterization of permissibility and a characterization of iterated admissibility. Since the correspondence between models with complete information and incomplete information are basically the same with the previous group, here the results are given without examples.

Theorem 4,3 (An alternative characterization of permissibility). Consider a finite 2-person static game $\Gamma=\left(C_{i}, u_{i}\right)_{i \in I}$ and the corresponding game form $G=\left(C_{i}\right)_{i \in I}$.

Then, $c_{i}^{*} \in C_{i}$ is permissible in $M^{c o}$ if and only if there is some finite lexicographic epistemic model $M^{i n}=\left(\Theta_{i}, w_{i}, \beta_{i}\right)_{i \in I}$ with incomplete information for $G$ and some $\theta_{i}^{*} \in \Theta_{i}$ with $w_{i}\left(\theta_{i}^{*}\right)=u_{i}$ such that

(a) $c_{i}^{*}$ is rational for $\theta_{i}^{*}$, and,

(b) $\theta_{i}^{*}$ expresses common full belief in caution, rationality, and primary belief in $u$.

Theorem 4,4 (Characterization of iterated admissibility). Consider a finite 2person static game $\Gamma=\left(C_{i}, u_{i}\right)_{i \in I}$ and the corresponding game form $G=\left(C_{i}\right)$.

Then $c_{i}^{*} \in C_{i}$ is iteratively admissible if and only if there is some finite epistemic model $M^{i n}=\left(\Theta_{i}, w_{i}, \beta_{i}\right)_{i \in I}$ with incomplete information for $G$ and some $\theta_{i}^{*} \in \Theta_{i}$ with $w_{i}\left(\theta_{i}^{*}\right)=u_{i}$ such that

(a) $c_{i}^{*}$ is rational for $\theta_{i}^{*}$, and

(b) $\theta_{i}^{*}$ expresses common full belief in caution, rationality, and common assumption of prior $u$ and that every good choice is supported.

\subsection{Proof of Theorem 4}

To show the only-if part of Theorem 4.1 (and that of all other theorems), we construct the following mapping from finite lexicographic epistemic models with complete information to those with incomplete information. Consider $\Gamma=\left(C_{i}, u_{i}\right)_{i \in I}$ and a finite lexicographic epistemic model $M^{c o}=\left(T_{i}, b_{i}\right)_{i \in I}$ with complete information for $\Gamma$. We first define types in a model with incomplete information in the following two steps:

Step 1. For each $i \in I$ and $t_{i} \in T_{i}$, let $\Pi_{i}\left(t_{i}\right)=\left(C_{i 1}, \ldots, C_{i L}\right)$ be the sequence of equivalence classes of choices in $C_{i}$ arranged from the most preferred to the least preferred under $t_{i}$. By Lemma 3 1 , for each $C_{i \ell}$ there is some $v_{i \ell}\left(t_{i}\right) \in V_{i}$ such that each choice in $C_{i \ell}$ is rational at $v_{i \ell}\left(t_{i}\right)$ under $t_{i}$, and $0=d\left(v_{i 1}\left(t_{i}\right), u_{i}\right)<d\left(v_{i 2}\left(t_{i}\right), u_{i}\right)<\ldots<$ $d\left(v_{i L}\left(t_{i}\right), u_{i}\right)$.

Step 2. We define $\Theta_{i}\left(t_{i}\right)=\left\{\theta_{i 1}\left(t_{i}\right), \ldots, \theta_{i L}\left(t_{i}\right)\right\}$ where for each $\ell=1, \ldots, L$, the type $\theta_{i \ell}\left(t_{i}\right)$ satisfies that (1) $w_{i}\left(\theta_{i \ell}\left(t_{i}\right)\right)=v_{i \ell}\left(t_{i}\right)$, and (2) $\beta_{i}\left(\theta_{i \ell}\left(t_{i}\right)\right)$ is obtained from $b_{i}\left(t_{i}\right)$ by replacing every $\left(c_{j}, t_{j}\right)$ with $c_{j} \in C_{j r} \in \Pi_{j}\left(t_{j}\right)$ for some $r$ with $\left(c_{j}, \theta_{j}\right)$ where $\theta_{j}=\theta_{j r}\left(t_{j}\right)$, that is, $w_{j}\left(\theta_{j}\right)$ is the utility function among those corresponding to $\Pi_{j}\left(t_{j}\right)$ in which $c_{j}$ is the rational for $t_{i}$.

For each $i \in I$, let $\Theta_{i}=\cup_{t_{i} \in T_{i}} \Theta_{i}\left(t_{i}\right)$. Here we have constructed a finite lexicographic epistemic model $M^{i n}=\left(\Theta_{i}, w_{i}, \beta_{i}\right)_{i \in I}$ for the corresponding game form $G=$ $\left(C_{i}\right)_{i \in I}$ with incomplete information. It should be noted that $M^{i n}$ is not uniquely determined since there are multiple sequences of utility functions satisfying the conditions in Lemma 3 . 1 . 
Table 9 The game for Example 42

\begin{tabular}{lll}
\hline$u_{1} \backslash u_{2}$ & $C$ & $D$ \\
\hline$A$ & 1,0 & 0,1 \\
$B$ & 0,0 & 0,1 \\
\hline
\end{tabular}

Table 10 Utility functions of player 1 corresponding to $t_{1}$ and $t_{2}$

\begin{tabular}{lllllll}
\cline { 1 - 3 } \cline { 5 - 6 }$v_{12}\left(t_{1}\right)$ & $C$ & $D$ & & $v_{22}\left(t_{2}\right)$ & $C$ & $D$ \\
\cline { 1 - 2 } \cline { 5 - 7 } & 1 & 0 & & 2 & 1 \\
$B$ & 0 & 1 & & 0 & 1 \\
\hline
\end{tabular}

We show how this construction works by the following example.

Example 42. Consider the game $\Gamma$ in Table 9 (Perea, 2012, p.188) and the lexicographic epistemic model $M^{c o}=\left(T_{i}, b_{i}\right)_{i \in I}$ of $\Gamma$ where $T_{1}=\left\{t_{1}\right\}, T_{2}=\left\{t_{2}\right\}$, and

$$
b_{1}\left(t_{1}\right)=\left(\left(D, t_{2}\right),\left(C, t_{2}\right)\right), b_{2}\left(t_{2}\right)=\left(\left(A, t_{1}\right),\left(B, t_{1}\right)\right) \text {. }
$$

We show how to construct a corresponding model $M^{i n}=\left(\Theta_{i}, w_{i}, \beta_{i}\right)_{i \in I}$. First, by Step 1 it can be seen that $\Pi_{1}\left(t_{1}\right)=(\{A\},\{B\})$ and $\Pi_{2}\left(t_{2}\right)=(\{D\},\{C\})$. We let $v_{11}\left(t_{1}\right)=u_{1}$ where $A$ is rational for $t_{1}$ and $v_{12}\left(t_{1}\right)$ where $B$ is rational for $t_{1}$ as follows. Similarly, we let $v_{21}\left(t_{2}\right)=u_{2}$ where $D$ is rational under $t_{2}$ and $v_{22}\left(t_{2}\right)$ where $C$ is rational under $t_{2}$ as follows:

Then we go to Step 2. It can be seen that $\Theta_{1}\left(t_{1}\right)=\left\{\theta_{11}\left(t_{1}\right), \theta_{12}\left(t_{1}\right)\right\}$, where

$$
\begin{aligned}
& w_{1}\left(\theta_{11}\left(t_{1}\right)\right)=v_{11}\left(t_{1}\right), \beta_{1}\left(\theta_{11}\left(t_{1}\right)\right)=\left(\left(D, \theta_{21}\left(t_{2}\right)\right),\left(C, \theta_{22}\left(t_{2}\right)\right)\right), \\
& w_{1}\left(\theta_{12}\left(t_{1}\right)\right)=v_{12}\left(t_{1}\right), \beta_{1}\left(\theta_{12}\left(t_{1}\right)\right)=\left(\left(D, \theta_{21}\left(t_{2}\right)\right),\left(C, \theta_{22}\left(t_{2}\right)\right)\right) .
\end{aligned}
$$

Also, $\Theta_{2}\left(t_{2}\right)=\left\{\theta_{21}\left(t_{2}\right), \theta_{22}\left(t_{2}\right)\right\}$, where

$$
\begin{aligned}
& w_{2}\left(\theta_{21}\left(t_{2}\right)\right)=v_{21}\left(t_{2}\right), \beta_{2}\left(\theta_{21}\left(t_{2}\right)\right)=\left(\left(A, \theta_{11}\left(t_{1}\right)\right),\left(B, \theta_{12}\left(t_{1}\right)\right)\right), \\
& w_{2}\left(\theta_{22}\left(t_{2}\right)\right)=v_{22}\left(t_{2}\right), \beta_{2}\left(\theta_{22}\left(t_{2}\right)\right)=\left(\left(A, \theta_{11}\left(t_{1}\right)\right),\left(B, \theta_{12}\left(t_{1}\right)\right)\right) .
\end{aligned}
$$

Consider $M^{c o}=\left(T_{i}, b_{i}\right)_{i \in I}$ and $M^{i n}=\left(\Theta_{i}, w_{i}, \beta_{i}\right)_{i \in I}$ corresponding to $M^{c o}$ constructed by the two steps above. We have the following observations.

Observation 411 (Redundancy). For each $t_{i} \in T_{i}$ and each $\theta_{i}, \theta_{i}^{\prime} \in \Theta_{i}\left(t_{i}\right), \beta_{i}\left(\theta_{i}\right)=$ $\beta_{i}\left(\theta_{i}^{\prime}\right)$

Observation 4,2 (Rationality). Each $\theta_{i} \in \Theta_{i}\left(t_{i}\right)$ believes in $j$ 's rationality.

Observation 4,3 (A better choice is supported by utilities nearer to $u$ ). Each $\theta_{i} \in \Theta_{i}\left(t_{i}\right)$ believes that a better choice is supported by utilities nearer to $u$.

The observations are true by construction. Observation 41 means that the difference between any two types in a $\Theta_{i}\left(t_{i}\right)$ is in the utility functions assigned to them. Observation 42 means that in an incomplete information model constructed from one with complete information, each type believes in the opponent's rationality. This is because in the construction, we requires that for each pair $\left(c_{j}, t_{j}\right)$ occurring in 
a belief, its counterpart in the incomplete information replaces $t_{j}$ by type in $\Theta_{j}\left(t_{j}\right)$ with the utility function in which $c_{j}$ is optimal for $t_{j}$. It follows from Observation 42 that each $\theta_{i} \in \Theta_{i}\left(t_{i}\right)$ expresses common full belief in rationality. Observation 43 implies that the best choice is supported by utilities nearest to $u$. It follows that each $\theta_{i} \in \Theta_{i}\left(t_{i}\right)$ expresses common full belief in that a best (better) choice is supported by utilities nearest (nearer) to $u$.

By construction, each $t_{i}$ shares the same belief about $j$ 's choices at each level with each $\theta_{i} \in \Theta_{i}\left(t_{i}\right)$; also, for each $t_{i} \in T_{i}$, the utility function assigned to $\theta_{i 1}\left(t_{i}\right)$ is $u_{i}$. It is clear that any $c_{i}$ rational for $t_{i}$ is also rational for $\theta_{i 1}\left(t_{i}\right)$. Therefore, to show the only-if part of Theorem 41 , we show that if $t_{i}$ expresses common full belief in caution and primary belief in rationality, then $\theta_{i 1}\left(t_{i}\right)$ expresses common belief in caution, primary belief in utilities nearest to $u$, and that a best choice is supported by utilities nearest to $u$.

Lemma 4, 1 (Caution $^{c o} \rightarrow$ Caution $\left.^{i n}\right)$. Consider $M^{c o}=\left(T_{i}, b_{i}\right)_{i \in I}$ and $M^{i n}=\left(\Theta_{i}, w_{i}, \beta_{i}\right)_{i \in I}$ corresponding to $M^{c o}$. If $t_{i} \in T_{i}$ expresses common full belief in caution, so does each $\theta_{i} \in \Theta_{i}\left(t_{i}\right)$.

Lemma 4,2 (Primary belief in rationality $\rightarrow$ primary belief in utilities nearest to $u$ ). Consider $M^{c o}=\left(T_{i}, b_{i}\right)_{i \in I}$ and $M^{i n}=\left(\Theta_{i}, w_{i}, \beta_{i}\right)_{i \in I}$ corresponding to $M^{c o}$. If $t_{i} \in T_{i}$ expresses common full belief in primary belief in rationality, then each $\theta_{i} \in \Theta_{i}\left(t_{i}\right)$ expresses common full belief in primary belief in utilities nearest to $u$.

Proof of the only-if part of Theorem 4. 1. Consider $M^{c o}=\left(T_{i}, b_{i}\right)_{i \in I}, M^{i n}=\left(\Theta_{i}, w_{i}, \beta_{i}\right)_{i \in I}$ corresponding to $M^{c o}$, a permissible choice $c_{i}^{*} \in C_{i}$, and $t_{i}^{*} \in T_{i}$ which is a type expressing common full belief in caution and primary belief in rationality such that $c_{i}^{*}$ is rational for $t_{i}^{*}$. Let $\theta_{i}^{*}=\theta_{i 1}\left(t_{i}^{*}\right)$. By definition, $w_{i}\left(\theta_{i}^{*}\right)=u_{i}$ and $\beta_{i}\left(\theta_{i}^{*}\right)$ has the same distribution on $j$ 's choices at each level as $b_{i}\left(t_{i}^{*}\right)$. Hence $c_{i}^{*}$ is rational for $\theta_{i}^{*}$. Also, it follows from Observation 4,3 and Lemmas 4,1 and 42 that $\theta_{i}^{*}$ expresses common full belief in caution, primary belief in utilities nearest to $u$, and that a best choice is supported by utilities nearest to $u$.

To show the if part, we need a mapping from models with incomplete information to those with complete information. Consider a finite 2-person static game $\Gamma=\left(C_{i}, u_{i}\right)_{i \in I}$, the corresponding game form $G=\left(C_{i}\right)_{i \in I}$, and a finite epistemic model $M^{i n}=\left(\Theta_{i}, w_{i}, \beta_{i}\right)_{i \in I}$ for $G$ with incomplete information. We construct a model $M^{c o}=\left(T_{i}, b_{i}\right)_{i \in I}$ for $\Gamma$ with complete information as follows. For each $\theta_{i} \in \Theta_{i}$, we define $E_{i}\left(\theta_{i}\right)=\left\{\theta_{i}^{\prime} \in \Theta_{i}: \beta_{i}\left(\theta_{i}^{\prime}\right)=\beta\left(\theta_{i}\right)\right\}$. In this way $\Theta_{i}$ is partitioned into several equivalence classes $\mathbb{E}_{i}=\left\{E_{i 1}, \ldots, E_{i L}\right\}$ where for each $\ell=1, . ., L, E_{i \ell}=E_{i}\left(\theta_{i}\right)$ for some $\theta_{i} \in \Theta_{i}$. To each $E_{i} \in \mathbb{E}_{i}$ we use $t_{i}\left(E_{i}\right)$ to denote a type. We define $b_{i}\left(t_{i}\left(E_{i}\right)\right)$ to be a lexicographic belief which is obtained from $\beta_{i}\left(\theta_{i}\right)$ by replacing each occurrence of $\left(c_{j}, \theta_{j}\right)$ by $\left(c_{j}, t_{j}\left(E_{j}\left(\theta_{j}\right)\right)\right)$; in other words, $b_{i}\left(t_{i}\left(E_{i}\right)\right)$ has the same distribution on choices at each level as $\beta_{i}\left(\theta_{i}\right)$ for each $\theta_{i} \in E_{i}$, while each $\theta_{j} \in \Theta_{j}\left(\theta_{i}\right)$ is replaced by $t_{j}\left(E_{j}\left(\theta_{j}\right)\right)$. For each $i \in I$, let $T_{i}=\left\{t_{i}\left(E_{i}\right)\right\}_{E_{i} \in \mathbb{E}_{i}}$. We have constructed from $M^{i n}$ a finite epistemic model $M^{c o}=\left(T_{i}, b_{i}\right)_{i \in I}$ with complete information for $\Gamma$.

We show how this construction works by the following example.

Example 43. Consider the game $\Gamma$ in Example 4, 2 and the model $M^{i n}=\left(\Theta_{i}, w_{i}, \beta_{i}\right)_{i \in I}$ 
for the corresponding game form where $\Theta_{1}=\left\{\theta_{11}, \theta_{12}\right\}, \Theta_{2}=\left\{\theta_{21}, \theta_{22}\right\}$, and

$$
\begin{aligned}
& w_{1}\left(\theta_{11}\right)=u_{1}, \beta_{1}\left(\theta_{11}\right)=\left(\left(D, \theta_{21}\right),\left(C, \theta_{22}\right)\right), \\
& w_{1}\left(\theta_{12}\right)=v_{1}, \beta_{1}\left(\theta_{12}\right)=\left(\left(D, \theta_{21}\right),\left(C, \theta_{22}\right)\right), \\
& w_{2}\left(\theta_{21}\right)=u_{2}, \beta_{2}\left(\theta_{21}\right)=\left(\left(A, \theta_{11}\right),\left(B, \theta_{12}\right)\right), \\
& w_{2}\left(\theta_{22}\right)=v_{2}, \beta_{2}\left(\theta_{22}\right)=\left(\left(A, \theta_{11}\right),\left(B, \theta_{12}\right)\right) .
\end{aligned}
$$

where $v_{1}=v_{12}\left(t_{1}\right)$ and $v_{2}=v_{22}\left(t_{2}\right)$ in Example 42 . It can be seen that $\mathbb{E}_{1}=\left\{\left\{\theta_{11}, \theta_{12}\right\}\right\}$ since $\beta_{1}\left(\theta_{11}\right)=\beta_{1}\left(\theta_{12}\right)$ and $\mathbb{E}_{2}=\left\{\left\{\theta_{21}, \theta_{22}\right\}\right\}$ since $\beta_{2}\left(\theta_{21}\right)=\beta_{2}\left(\theta_{22}\right)$. Corresponding to those equivalence classes we have $t_{1}\left(\left\{\theta_{11}, \theta_{12}\right\}\right)$ and $t_{2}\left(\left\{\theta_{21}, \theta_{22}\right\}\right)$, and

$$
\begin{aligned}
& b_{1}\left(t_{1}\left(\left\{\theta_{11}, \theta_{12}\right\}\right)\right)=\left(\left(D, t_{2}\left(\left\{\theta_{21}, \theta_{22}\right\}\right)\right),\left(C, t_{2}\left(\left\{\theta_{21}, \theta_{22}\right\}\right)\right)\right), \\
& b_{2}\left(t_{2}\left(\left\{\theta_{21}, \theta_{22}\right\}\right)\right)=\left(\left(A, t_{1}\left(\left\{\theta_{11}, \theta_{12}\right\}\right)\right),\left(B, t_{1}\left(\left\{\theta_{11}, \theta_{12}\right\}\right)\right)\right) .
\end{aligned}
$$

It can be seen that this is the reversion of the previous construction if each type has distinct belief on choices of the opposite. Indeed, let $M^{c o}=\left(T_{i}, b_{i}\right)_{i \in I}$ satisfying that $b_{i}\left(t_{i}\right) \neq b_{i}\left(t_{i}^{\prime}\right)$ for each $t_{i}, t_{i}^{\prime} \in T_{i}$ with $t_{i} \neq t_{i}^{\prime}$, and $M^{i n}=\left(\Theta_{i}, w_{i}, \beta_{i}\right)_{i \in I}$ be constructed from $M^{c o}$ by the previous two steps. Then $\mathbb{E}_{i}=\left\{\Theta_{i}\left(t_{i}\right)\right\}_{t_{i} \in T_{i}}$ and $t_{i}\left(\Theta_{i}\left(t_{i}\right)\right)=t_{i}$ for each $i \in I$.

To show the if part of Theorem 41 , we need the following lemmas.

Lemma 43 (Caution $^{i n} \rightarrow$ Caution $\left.^{c o}\right)$. Consider $M^{i n}=\left(\Theta_{i}, w_{i}, \beta_{i}\right)_{i \in I}$ and $M^{c o}=$ $\left(T_{i}, b_{i}\right)_{i \in I}$ corresponding to $M^{\text {in }}$ constructed by the above approach. If $\theta_{i} \in \Theta_{i}$ expresses common full belief in caution, so does $t_{i}\left(E_{i}\left(\theta_{i}\right)\right)$.

Lemma 4,4 (Caution ${ }^{i n}+$ primary belief in utilities nearest to $u+$ a best choice is supported by utilities nearest to $u \rightarrow$ Primary belief in rationality). Consider $M^{i n}=\left(\Theta_{i}, w_{i}, \beta_{i}\right)_{i \in I}$ and $M^{c o}=\left(T_{i}, b_{i}\right)_{i \in I}$ corresponding to $M^{i n}$. If $\theta_{i} \in \Theta_{i}$ expresses common full belief in caution, primary belief in utilities nearest to $u$, and that a best choice is supported by utilities nearest to $u$, then $t_{i}\left(E_{i}\left(\theta_{i}\right)\right)$ expresses common full belief in primary belief in rationality.

Proof of the if part of Theorem 4 1. Consider $M^{c o}=\left(T_{i}, b_{i}\right)_{i \in I}$ corresponding to $M^{\text {in }}$ and $c_{i}^{*} \in C_{i}$ rational for some $\theta_{i}^{*}$ with $w_{i}\left(\theta_{i}^{*}\right)=u_{i}$ which expresses common full belief in caution, primary belief in utilities nearest to $u$, and that a best choice is supported by utilities nearest to $u$. Consider $t_{i}\left(E_{i}\left(\theta_{i}^{*}\right)\right)$. Since $w_{i}\left(\theta_{i}^{*}\right)=u_{i}$ and $b_{i}\left(t_{i}\left(E_{i}\left(\theta_{i}^{*}\right)\right)\right)$ has the same distribution on $j$ 's choices at each level as $\beta_{i}\left(\theta_{i}^{*}\right), c_{i}^{*}$ is rational for $t_{i}\left(E_{i}\left(\theta_{i}^{*}\right)\right)$. Also, by Lemmas 43 and $44, t_{i}\left(E_{i}\left(\theta_{i}^{*}\right)\right)$ expresses common full belief in caution and primary belief in rationality. Hence $c_{i}^{*}$ is permissible in $\Gamma$.

\subsection{Proof of Theorem 4,2}

To show the only-if part of Theorem 42 , we need the following lemmas.

Lemma 4,5 (Respect of preferences $\rightarrow u$-centered belief). Consider $M^{c o}=\left(T_{i}, b_{i}\right)_{i \in I}$ and $M^{i n}=\left(\Theta_{i}, w_{i}, \beta_{i}\right)_{i \in I}$ corresponding to $M^{c o}$ constructed by the two steps in Section 43. If $t_{i} \in T_{i}$ expresses common full belief in caution and respect of preferences, 
then each $\theta_{i} \in \Theta_{i}\left(t_{i}\right)$ expresses full belief in $u$-centered belief.

Proof of the only-if part of Theorem 4, 2. Consider $M^{c o}=\left(T_{i}, b_{i}\right)_{i \in I}, M^{i n}=\left(\Theta_{i}, w_{i}, \beta_{i}\right)_{i \in I}$ corresponding to $M^{c o}$, a properly rationalizable $c_{i}^{*} \in C_{i}$, and $t_{i}^{*} \in T_{i}$ expressing common full belief in caution and respect of preferences such that $c_{i}^{*}$ is rational for $t_{i}^{*}$. Let $\theta_{i}^{*}=\theta_{i 1}\left(t_{i}^{*}\right)$. Since $w_{i}\left(\theta_{i}^{*}\right)=u_{i}$ and $\beta_{i}\left(\theta_{i}^{*}\right)$ has the same distribution on $j$ 's choices as $b_{i}\left(t_{i}^{*}\right), c_{i}^{*}$ is rational for $\theta_{i}^{*}$. Also, it follows from Observations 43 and Lemmas 41 and 45 that $\theta_{i}^{*}$ expresses common belief in caution, $u$-centered belief, and that a better choice is supported by utilities nearer to $u$.

To show the if part, we need the following lemma.

Lemma 4,6 (Caution $^{i n}+u$-centered belief + a better choice is supported by utilities nearer to $u \rightarrow$ respect of preferences). Consider $M^{i n}=\left(\Theta_{i}, w_{i}, \beta_{i}\right)_{i \in I}$ and $M^{c o}=\left(T_{i}, b_{i}\right)_{i \in I}$ corresponding to $M^{i n}$ constructed by the approach in Section 43 . If $\theta_{i} \in \Theta_{i}$ expresses common full belief in caution, $u$-centered belief, and that a better choice is supported by utilities nearer to $u$, then $t_{i}\left(E_{i}\left(\theta_{i}\right)\right)$ expresses common full belief in respect of preferences.

Proof of the if part of Theorem 4,2. Consider $M^{c o}=\left(T_{i}, b_{i}\right)_{i \in I}$ corresponding to $M^{\text {in }}$ and $c_{i}^{*} \in C_{i}$ be rational for some $\theta_{i}^{*}$ with $w_{i}\left(\theta_{i}^{*}\right)=u_{i}$ which expresses common belief in caution, rationality, $u$-centered belief, and that a better choice is supported by utilities nearer to $u$. Consider $t_{i}\left(E_{i}\left(\theta_{i}^{*}\right)\right)$. Since $w_{i}\left(\theta_{i}^{*}\right)=u_{i}$ and $t_{i}\left(E_{i}\left(\theta_{i}^{*}\right)\right)$ and $\theta_{i}^{*}$ have the same distribution on $j$ 's choices in each level, $c_{i}^{*}$ is rational for $t_{i}\left(E_{i}\left(\theta_{i}^{*}\right)\right)$. Also, it follows from Lemmas 43 and 46 that $t_{i}\left(E_{i}\left(\theta_{i}^{*}\right)\right)$ expresses common full belief in caution and respect of preferences. Hence $c_{i}^{*}$ is properly rationalizable in $\Gamma$.

\subsection{Proof of Theorem 43}

The only-if part of Theorem 43 follows directly from Observation 42, Lemma 4 1, and the following lemma whose proof can be found in Section 6 .

Lemma 4,7 (Primary belief in rationality $\rightarrow$ Primary belief in $u$ ). Consider $M^{c o}=$ $\left(T_{i}, b_{i}\right)_{i \in I}$ and $M^{i n}=\left(\Theta_{i}, w_{i}, \beta_{i}\right)_{i \in I}$ corresponding to $M^{c o}$. If $t_{i} \in T_{i}$ expresses common full belief in primary belief in rationality, then each $\theta_{i} \in \Theta_{i}\left(t_{i}\right)$ expresses common full belief in primary belief in $u$.

To show the if part, we need the following lemma.

Lemma 48 (Rationality + primary belief in $u \rightarrow$ Primary belief in rationality). Consider $M^{i n}=\left(\Theta_{i}, w_{i}, \beta_{i}\right)_{i \in I}$ and $M^{c o}=\left(T_{i}, b_{i}\right)_{i \in I}$ corresponding to $M^{i n}$. If $\theta_{i} \in \Theta_{i}$ expresses common full belief in rationality and primary belief in $u$, then $t_{i}\left(E_{i}\left(\theta_{i}\right)\right)$ expresses common full belief in primary belief in rationality.

Proof of the if part of Theorem 4,3. Consider $M^{c o}=\left(T_{i}, b_{i}\right)_{i \in I}$ corresponding to $M^{\text {in }}$ and $c_{i}^{*} \in C_{i}$ rational for some $\theta_{i}^{*}$ with $w_{i}\left(\theta_{i}^{*}\right)=u_{i}$ which expresses common full belief in caution, rationality, and primary belief in $u$. Consider $t_{i}\left(E_{i}\left(\theta_{i}^{*}\right)\right)$. Since $w_{i}\left(\theta_{i}^{*}\right)=u_{i}$ and $b_{i}\left(t_{i}\left(E_{i}\left(\theta_{i}^{*}\right)\right)\right)$ has the same distribution on $j$ 's choices at each level as $\beta_{i}\left(\theta_{i}^{*}\right), c_{i}^{*}$ is rational for $t_{i}\left(E_{i}\left(\theta_{i}^{*}\right)\right)$. By Lemmas 43 and $48, t_{i}\left(E_{i}\left(\theta_{i}^{*}\right)\right)$ expresses common full belief in caution and primary belief in rationality. Hence $c_{i}^{*}$ is permissible in $\Gamma$. 
4.6 Proof of Theorem 44

Lemma 4,9 (Assumption of rationality $\rightarrow$ every good choice is supported + prior assumption of $u$, every good choice is supported + prior assumption of $u+$ rationality $\rightarrow$ assumption of rationality). Consider $M^{c o}=\left(T_{i}, b_{i}\right)_{i \in I}$ and $M^{i n}=$ $\left(\Theta_{i}, w_{i}, \beta_{i}\right)_{i \in I}$ corresponding to $M^{c o}$. If $t_{i} \in T_{i}$ expresses common assumption of rationality, then each $\theta_{i} \in \Theta_{i}\left(t_{i}\right)$ expresses common assumption of prior $u$ and that every good choice is supported.

On the other hand, consider $M^{i n}=\left(\Theta_{i}, w_{i}, \beta_{i}\right)_{i \in I}$ and $M^{c o}=\left(T_{i}, b_{i}\right)_{i \in I}$ corresponding to $M^{i n}$. If $\theta_{i} \in \Theta_{i}$ expresses common full belief in rationality and common assumption of prior $u$ and that every good choice is supported, then $t_{i}\left(E_{i}\left(\theta_{i}\right)\right)$ expresses common assumption of rationality.

Here we have to combine the two ways (i.e., complete information models to incomplete information ones and the other way around) into one lemma. The reason is that to prove Lemma 4, 9 , we cannot separate them as we did in the previous lemmas; we need to show two ways in the induction base as well as in the induction step. The details are left to Section 6 .

Proof of Theorem 44. (Only-if) Consider $M^{c o}=\left(T_{i}, b_{i}\right)_{i \in I}, M^{i n}=\left(\Theta_{i}, w_{i}, \beta_{i}\right)_{i \in I}$ corresponding to $M^{c o}$, an iteratively admissible choice $c_{i}^{*} \in C_{i}$, and $t_{i}^{*} \in T_{i}$ expressing common full belief in caution and common assumption of rationality such that $c_{i}^{*}$ is rational for $t_{i}^{*}$. Let $\theta_{i}^{*}=\theta_{i 1}\left(t_{i}^{*}\right)$. By definition, $w_{i}\left(\theta_{i}^{*}\right)=u_{i}$ and $\beta_{i}\left(\theta_{i}^{*}\right)$ has the same distribution on $j$ 's choices at each level as $b_{i}\left(t_{i}^{*}\right)$. Hence $c_{i}^{*}$ is rational for $\theta_{i}^{*}$. Also, it follows from Observation 4, 2 and Lemmas 4, 1 and 4,9 that $\theta_{i}^{*}$ expresses common full belief in caution, rationality, and common assumption of prior $u$ and that a good choice is supported.

(If). Consider $M^{c o}=\left(T_{i}, b_{i}\right)_{i \in I}$ corresponding to $M^{i n}$ and $c_{i}^{*} \in C_{i}$ rational for some $\theta_{i}^{*}$ with $w_{i}\left(\theta_{i}^{*}\right)=u_{i}$ which expresses common full belief in caution, rationality, and common assumption of prior $u$ and that a good choice is supported. Consider $t_{i}\left(E_{i}\left(\theta_{i}^{*}\right)\right)$. Since $w_{i}\left(\theta_{i}^{*}\right)=u_{i}$ and $b_{i}\left(t_{i}\left(E_{i}\left(\theta_{i}^{*}\right)\right)\right)$ has the same distribution on $j$ 's choices at each level as $\beta_{i}\left(\theta_{i}^{*}\right), c_{i}^{*}$ is rational for $t_{i}\left(E_{i}\left(\theta_{i}^{*}\right)\right)$. By Lemmas 42 and $49, t_{i}\left(E_{i}\left(\theta_{i}^{*}\right)\right)$ expresses common full belief in caution and common assumption of rationality. Therefore, $c_{i}^{*}$ is iteratively admissible.

\section{Concluding Remarks}

\subsection{Faithful parallel to Perea and Roy (2017)'s Theorem 6.1}

All theorems here can be rephrased as faithful parallels to Perea and Roy (2017)'s Theorem 6.1, focusing on equivalence between belief hierarchies in complete and incomplete information models. We adopt the forms here because the coincidence of belief hierarchies holds by construction, and we think it is unnecessary to mention it independently.

Also, our proofs are based on constructing a specific correspondence between two models. It can be seen that this correspondence can be translated directly into probabilistic models and be used to show Perea and Roy (2017)'s Theorem 6.1. Further, 
it can be seen that, by using our Lemma 1 , belief in rationality under closest utility function in Perea and Roy (2017) can be replaced by the weaker one (Definition 34 (4.2)) here.

\subsection{Extending to $n$-person cases}

Both Perea and Roy (2017) and this paper focus on 2-person games. To extend those results to $n$-person cases, the problem is how to define the distance between utility functions and how to relate the distance with the locations of choice-type pairs. In a 2-person game, a type of $i$ only needs to consider distributions on $\Delta\left(C_{j} \times \Theta_{j}\right)$. Hence a "cell" in $\beta_{i}\left(\theta_{i}\right)$ is just a pair $\left(c_{j}, \theta_{j}\right)$, and its location in $\beta_{i}\left(\theta_{i}\right)$ can be related directly to the distance $d\left(w_{j}\left(\theta_{j}\right), u_{j}\right)$. In contrast, in an $n$-person case a "cell" of a lexicographic belief contains $n-1$ pairs such as

$$
\left\langle\left(c_{1}, \theta_{1}\right), \ldots,\left(c_{i-1}, \theta_{i-1}\right),\left(c_{i+1}, \theta_{i+1}\right), \ldots,\left(c_{n}, \theta_{n}\right)\right\rangle,
$$

and consequently there are $n-1$ distances, i.e.,

$$
d\left(w_{1}\left(\theta_{1}\right), u_{1}\right), \ldots, d\left(w_{i-1}\left(\theta_{i-1}\right), u_{i-1}\right), d\left(w_{i+1}\left(\theta_{i+1}\right), u_{i+1}\right), \ldots, d\left(w_{n}\left(\theta_{n}\right), u_{n}\right) .
$$

Then the problem is how to connect the location of this cell and those distances. We believe that the results of Perea and Roy (2017) and this paper can be extended to $n$ person games with a proper definition of the distances and their relation with locations of "cells" in lexicographic beliefs. Further work is expected in that direction.

\subsection{The role of rationality}

Rationality has not been used in Theorems 4,1 and 4,2 even though in epistemic models with incomplete information constructed in Section 4/3 each type has a common full belief in rationality (Observation 4 2). On the other hand, there are also epistemic models with types satisfying all conditions in Theorems 4,1 and 4,2 but not believing in rationality. Here is an example.

Example 5,1 (Rationality is not satisfied). Consider the game $\Gamma$ in Example 4.1 and the lexicographic epistemic model $M^{i n}=\left(\Theta_{i}, w_{i}, \beta_{i}\right)_{i \in I}$ with incomplete information for the corresponding game form where $\Theta_{1}=\left\{\theta_{11}, \theta_{12}, \theta_{13}\right\}, \Theta_{2}=\left\{\theta_{21}, \theta_{22}, \theta_{23}\right\}$, and

$$
\begin{aligned}
& w_{1}\left(\theta_{11}\right)=u_{1}, \beta_{1}\left(\theta_{11}\right)=\left(\left(D, \theta_{21}\right),\left(F, \theta_{22}\right),\left(E, \theta_{23}\right)\right), \\
& w_{1}\left(\theta_{12}\right)=v_{1}, \beta_{1}\left(\theta_{12}\right)=\left(\left(D, \theta_{21}\right),\left(F, \theta_{22}\right),\left(E, \theta_{23}\right)\right), \\
& w_{1}\left(\theta_{13}\right)=v_{1}^{\prime}, \beta_{1}\left(\theta_{13}\right)=\left(\left(D, \theta_{21}\right),\left(F, \theta_{22}\right),\left(E, \theta_{23}\right)\right), \\
& \left.w_{2}\left(\theta_{21}\right)=v_{2}, \beta_{2}\left(\theta_{21}\right)=\left(\left(C, \theta_{11}\right),\left(B, \theta_{12}\right)\right),\left(A, \theta_{13}\right)\right), \\
& \left.w_{2}\left(\theta_{22}\right)=v_{2}^{\prime}, \beta_{2}\left(\theta_{22}\right)=\left(\left(C, \theta_{11}\right),\left(B, \theta_{12}\right)\right),\left(A, \theta_{13}\right)\right), \\
& \left.w_{2}\left(\theta_{23}\right)=v_{2}^{\prime \prime}, \beta_{2}\left(\theta_{23}\right)=\left(\left(C, \theta_{11}\right),\left(B, \theta_{12}\right)\right),\left(A, \theta_{13}\right)\right) .
\end{aligned}
$$

where $v_{1}, v_{1}^{\prime}, v_{2}, v_{2}^{\prime}$ are the same as in Example 4.1 and $v_{2}^{\prime \prime}$ is illustrated in Table 11. 
Table 11 An alternative utility function for player 2

\begin{tabular}{cccc}
\hline$v_{2}^{\prime \prime}$ & $D$ & $E$ & $F$ \\
\hline$A$ & 3 & 2 & 1 \\
$B$ & 3 & 2 & 1 \\
$C$ & 6 & 4 & 5 \\
\hline
\end{tabular}

It can be seen that $\theta_{11}$ expresses common full belief in caution, $u$-centered belief and that a better choice is supported by utilities nearer to $u$ (therefore primary belief in utilities nearest to $u$ and that a best choice is supported by utilities nearest to $u$ are also satisfied) but not rationality since, for example, $D$ is not rational for $\theta_{21}$. However, consider the model $M^{c o}=\left(T_{i}, b_{i}\right)_{i \in I}$ for $\Gamma$ constructed from $M^{i n}$. Indeed, since $\mathbb{E}_{1}=$ $\left\{\left\{\theta_{11}, \theta_{12}, \theta_{13}\right\}\right\}$ and $\mathbb{E}_{2}=\left\{\left\{\theta_{21}, \theta_{22}, \theta_{23}\right\}\right\}$, by letting $t_{1}=t_{1}\left(\left\{\theta_{11}, \theta_{12}, \theta_{13}\right\}\right)$ and $t_{2}=t_{2}\left(\left\{\theta_{21}, \theta_{22}, \theta_{23}\right\}\right)$, we obtain $M^{c o}=\left(T_{i}, b_{i}\right)_{i \in I}$ for $\Gamma$ where $T_{1}=\left\{t_{1}\right\}, T_{2}=\left\{t_{2}\right\}$, and

$$
b_{1}\left(t_{1}\right)=\left(\left(D, t_{2}\right),\left(F, t_{2}\right),\left(E, t_{2}\right)\right), b_{2}\left(t_{2}\right)=\left(\left(C, t_{1}\right),\left(B, t_{1}\right),\left(A, t_{1}\right)\right) .
$$

It can be seen that $t_{1}$ expresses caution and common full belief in respect of preferences (therefore primary belief in rationality). Further, $C$ is optimal for both $\theta_{11}$ and $t_{1}$.

On the other hand, rationality plays a critical role in the characterizations of the second group. It seems that whether or not using rationality in the characterization differentiates the two refinements of permissibility within the incomplete information framework, which corresponds to the fact that, in complete information models, there is no general relationship between respect of preferences and assumption of rationality (Perea, 2012). It would be interesting that any future research could confirm this statement or show that proper rationalizability can be characterized with rationality while iterated admissibility can be done without it.

On the other hand, as shown in the construction in Section 4 3 , it is always possible to construct epistemic models with incomplete information which satisfies rationality as well as all conditions in Theorems 4,1 and 42 . Further, prior belief in $u$ is a condition between primary belief in $u$ and $u$-centered belief. Those seem correspond to the fact within the complete information framework that there is always possible to construct belief hierarchy which both assumes the opponent's rationality and respects the opponent's preferences (Perea, 2012).

\subsection{An ordinal distance on $V_{i}$}

In this note, we use the Euclidean distance to measure similarity between utility functions. As mentioned in Section 31, the Euclidean distance is cardinal. We can define an ordinal distance as follows to replace it. Let $\beta_{i}$ be a lexicographic belief on $\Delta\left(S_{j} \times \Theta_{j}\right)$. For each $v_{i}, u_{i} \in V_{i}$, define $d^{\beta_{i}}\left(v_{i}, u_{i}\right)=\mid\left\{\left\{s_{i}, s_{i}^{\prime}\right\}: s_{i}, s_{i}^{\prime} \in S_{i}\right.$ and the preference between $s_{i}$ and $s_{i}^{\prime}$ under $\beta_{i}$ at $v_{i}$ are different from that at $\left.u_{i}\right\} \mid$. It can be seen that $d^{\beta_{i}}$ is a variation of Hamming distance (Hamming, 1950). It measures similarity between preferences under $\beta_{i}$ represented by $v_{i}$ and that by $u_{i}$, i.e., it measures the 
ordinal difference between $v_{i}$ and $u_{i}$. This does not belong to the group of distances characterized in Section 3.3 of Perea and Roy (2017) since there is no norm on $V_{i}$ to support $d^{\beta_{i}}$. Lemma 3. 1 still holds under $d^{\beta_{i}}$ since even if we replace $d$ by $d^{\beta_{i}}$ in Lemma 3.1 (c), the constructed utility function sequence in the proof still satisfies it. Hence $d$ in Definition 34 can be replaced by $d^{\beta_{i}}$ with appropriate $\beta_{i}$ and the characterization results still hold. Also, by replacing rationality under closest utility function by our Definition 34 (4.2), Perea and Roy (2017)'s Theorem 6.1 still holds under $d^{\beta_{i}}$.

\subsection{Weakening caution in Theorems 43 and 44}

Caution in Theorems 4, 3 and 4 4 can be replaced by a weaker concept called "weak caution" which is defined as follows.

Definition 5,1 (Weak caution). Consider a game form $G=\left(C_{i}\right)_{i \in I}$ and a lexicographic epistemic model $M^{i n}=\left(\Theta_{i}, w_{i}, \beta_{i}\right)_{i \in I}$ for $G$ with incomplete information. $\theta_{i} \in \Theta_{i}$ is weakly cautious iff for each $c_{j} \in C_{j}$, there is some $\theta_{j} \in \Theta_{j}$ such that $\theta_{i}$ deems $\left(c_{j}, \theta_{j}\right)$ possible.

Definition 5,1 is weaker than Definition 3, 2 since it only requires that each choice should appear in the belief of $\theta_{i}$ but does not require that it should be paired with each belief of $j$ deemed possible by $\theta_{i}$. Nevertheless, we will show in Lemma 51 that in with other conditions in this characterization it leads to caution.

Proposition 5.1 (An alternative characterization of permissibility). Consider a finite 2-person static game $\Gamma=\left(C_{i}, u_{i}\right)_{i \in I}$, the corresponding game form $G=\left(C_{i}\right)_{i \in I}$, and a finite lexicographic epistemic model $M^{c o}=\left(T_{i}, b_{i}\right)_{i \in I}$ for $\Gamma$.

Then, $c_{i}^{*} \in C_{i}$ is permissible in $M^{c o}$ if and only if there is some finite lexicographic epistemic model $M^{i n}=\left(\Theta_{i}, w_{i}, \beta_{i}\right)_{i \in I}$ with incomplete information for $G$ and some $\theta_{i}^{*} \in \Theta_{i}$ with $w_{i}\left(\theta_{i}^{*}\right)=u_{i}$ such that

(a) $c_{i}^{*}$ is rational for $\theta_{i}^{*}$, and,

(b) $\theta_{i}^{*}$ expresses common full belief in weak caution, rationality, and primary belief in $u$.

The only-if part holds since weak caution is weaker than caution. The if part needs first to show that weak caution is enough for the characterization. Here, we show that the corresponding concept in complete information model can replace caution and characterize permissibility. Then we can use the mapping between complete and incomplete information models constructed in Section 4 3. Let $M^{c o}=\left(T_{i}, b_{i}\right)_{i \in I}$ be a lexicographic model for $\Gamma=\left(C_{i}, u_{i}\right)_{i \in I}$ with complete information. $t_{i} \in T_{i}$ is weakly cautious iff for each $c_{j} \in C_{j}$, there is some $t_{j} \in T_{j}$ such that $t_{i}$ deems $\left(c_{j}, t_{j}\right)$ possible. We have the following lemma.

Lemma 5.1 (Characterizing permissibility by weak caution). Consider a lexicographic epistemic model $M^{c o}=\left(T_{i}, b_{i}\right)_{i \in I}$ for a game $\Gamma=\left(C_{i}, u_{i}\right)_{i \in I} . c_{i}^{*} \in C_{i}$ is permissible if and only if it is rational to some $t_{i}^{*} \in T_{i}$ which expresses common full belief in weak caution and primary belief in rationality.

Also, we need the following lemmas. 
Lemma5,2 $\left(\right.$ Weak caution ${ }^{i n} \rightarrow$ weak caution $\left.^{c o}\right)$. Let $M^{i n}=\left(\Theta_{i}, w_{i}, \beta_{i}\right)_{i \in I}$ and $M^{c o}=$ $\left(T_{i}, b_{i}\right)_{i \in I}$ be constructed from $M^{i n}$ by the above approach. If $\theta_{i} \in \Theta_{i}$ expresses common full belief in weak caution, so does $t_{i}\left(E_{i}\left(\theta_{i}\right)\right)$.

We omit the proof of Lemma 5,2 since it can be shown in a similar way as Lemma 4 3. It can be seen that Proposition 5. 1 follows directly from Lemmas 51 and 51 and Theorem 43. Similarly, it can be seen that caution in Theorem 44 can be replaced by weak caution.

However, it should be noted that caution cannot be weakened in Theorems 41 and 42 . For Theorem 4 1 , caution plays an important role in the proof of the if part; without it, primary belief in utilities nearest to $u$ and that a best choice is supported by utilities nearest to $u$ cannot imply primary belief in rationality. For Theorem 42 , the interpolation method used in the proof of Lemma 5.1 does not work in general since different types may have different orders there.

\section{Proofs}

Proof of Lemma 3,1. We construct a sequence satisfying (a)-(c) by induction. First, let $v_{i 1}=u_{i}$. Suppose that for some $\ell \in\{1, \ldots, L-1\}$ we have defined $v_{i 1}, \ldots, v_{i \ell}$ satisfying (a)-(c). Now we show how to define $v_{i, \ell+1}$. It can be seen that there exists $M_{\ell+1}>0$ such that $v_{i \ell}\left(c_{i, \ell+1}, \beta_{i 1}\right)+M_{\ell+1}>v_{i \ell}\left(c_{i \ell}, \beta_{i 1}\right)$ for all $c_{i \ell} \in C_{i \ell}$ and $c_{i, \ell+1} \in C_{i, \ell+1}$. We define $v_{i, \ell+1}$ as follows: for each $\left(c_{i}, c_{j}\right) \in C$,

$$
v_{i, \ell+1}\left(c_{i}, c_{j}\right)=\left\{\begin{array}{cc}
v_{i \ell}\left(c_{i}, c_{j}\right)+M_{\ell+1} & \text { if } c_{i} \in C_{i, \ell+1} \text { and } c_{j} \in \operatorname{supp} \beta_{i 1} \\
v_{i \ell}\left(c_{i}, c_{j}\right) & \text { otherwise }
\end{array}\right.
$$

It can be seen that each $c_{i, \ell+1} \in C_{i, \ell+1}$ is rational at $v_{i, \ell+1}$ under $\beta_{i}$. Also, since $d\left(v_{i, \ell+1}, v_{i \ell}\right)=\left(M_{\ell+1}^{2} \times\left|C_{i, \ell+1}\right| \times\left|\operatorname{supp} \beta_{i 1}\right|\right)^{1 / 2}>0, d\left(v_{i, \ell+1}, u_{i}\right)=d\left(v_{i, \ell+1}, v_{i \ell}\right)+d\left(v_{i n}, u_{i}\right)>$ $d\left(v_{i n}, u_{i}\right)$. By induction, we can obtain a sequence $v_{i 1}, \ldots, v_{i L} \in V_{i}$ satisfying (a)-(c).

It should be noted that, given $u_{i}$ and $\beta_{i}$, the sequence $v_{i 1}, \ldots, v_{i L}$ satisfying (a)(c) is not unique. The basic idea behind this inductive construction is depicted as follows. Suppose that $u_{i}\left(c_{i 1}, \beta_{i}\right)>u_{i}\left(c_{i 2}, \beta_{i}\right)>\ldots>u_{i}\left(c_{i N}, \beta_{i}\right)$, that is, $\Pi_{i}\left(\beta_{i}\right)=$ $\left(\left\{c_{i 1}\right\},\left\{c_{i 2}\right\}, \ldots,\left\{c_{i N}\right\}\right)$, then

$$
\left(c_{i 1}, c_{i 2}, c_{i 3}, \ldots, c_{i N}\right) \stackrel{v_{i 2}}{\longrightarrow}\left(c_{i 2}, c_{i 1}, c_{i 3}, \ldots, c_{i N},\right) \stackrel{v_{i 3}}{\longrightarrow}\left(c_{i 3}, c_{i 2}, c_{i 1} \ldots, c_{i N}\right) \ldots \underset{v_{i N}}{\longrightarrow}\left(c_{i N}, c_{i, N-1}, \ldots, c_{i 1}\right)
$$

Informally speaking, we take equivalent classes of choices one by one to the foremost location of the sequence according to the order of preference in $u_{i}$ under $\beta_{i}$. The following example shows how this construction works.

Example 6.1. Consider $u_{1}$ in Example 2]1. Under the lexicographic belief $\beta_{1}=$ $(D, E, F), A$ is preferred to $B$ and $B$ is preferred to $C$ in $u_{1}$, that is, $\Pi_{1}\left(\beta_{1}\right)=(\{A\},\{B\},\{C\})$. We can define $v_{11}, v_{12}$, and $v_{13}$ as in Table 12 .

At $v_{11}$, the order of preferences is $(A, B, C)$ under $\beta_{1}$, at $v_{12}$ it is $(B, A, C)$, and at $v_{13}$ it is $(C, B, A)$.

Proof of Lemma 4.1. We show this statement by induction. First we show that if $t_{i}$ is cautious, then each $\theta_{i} \in \Theta_{i}\left(t_{i}\right)$ is also cautious. Let $c_{j} \in C_{j}$ and $\theta_{j} \in \Theta_{j}\left(\theta_{i}\right)$. 
Table 12 Alternative utility functions for players 1 corresponding to $\Pi_{1}\left(\beta_{1}\right)$

\begin{tabular}{|c|c|c|c|c|c|c|c|c|c|c|c|c|c|}
\hline$v_{11}=u_{1}$ & $D$ & $E$ & $F$ & & $v_{12}$ & $D$ & $E$ & $F$ & & $v_{13}$ & $D$ & $E$ & $F$ \\
\hline$A$ & 1 & 1 & 1 & $\longrightarrow$ & $A$ & 1 & 1 & 1 & $\longrightarrow$ & $A$ & 1 & 1 & 1 \\
\hline$B$ & 1 & 1 & 0 & & $B$ & 2 & 1 & 0 & & $B$ & 2 & 1 & 0 \\
\hline$C$ & 1 & 0 & 1 & & $C$ & 1 & 0 & 1 & & $C$ & 3 & 0 & 1 \\
\hline
\end{tabular}

By construction, it can be seen that the type $t_{j} \in T_{j}$ satisfying the condition that $\theta_{j} \in \Theta_{j}\left(t_{j}\right)$ is in $T_{j}\left(t_{i}\right)$. Since $t_{i}$ is cautious, $t_{i}$ deems $\left(c_{j}, t_{j}\right)$ possible. Consider the pair $\left(c_{j}, \theta_{j}^{\prime}\right)$ in $\beta_{i}\left(\theta_{i}\right)$ corresponding to $\left(c_{j}, t_{j}\right)$. Since both $\theta_{j}$ and $\theta_{j}^{\prime}$ are in $\Theta_{j}\left(t_{j}\right)$, it follows from Observation 41 that $\beta_{j}\left(\theta_{j}\right)=\beta_{j}\left(\theta_{j}^{\prime}\right)$. Hence $\left(c_{j}, \theta_{j}^{w_{j}\left(\theta_{j}^{\prime}\right)}\right)$ is deemed possible by $\theta_{i}$. Here we have shown that $\theta_{i}$ is cautious.

Suppose we have shown that, for each $i \in I$, if $t_{i}$ expresses $n$-fold full belief in caution then so does each $\theta_{i} \in \Theta_{i}\left(t_{i}\right)$. Now suppose that $t_{i}$ expresses $(n+1)$-fold full belief in caution, i.e., each $t_{j} \in T_{j}\left(t_{i}\right)$ expresses $n$-fold full belief in caution. By construction, for each $\theta_{i} \in \Theta_{i}\left(t_{i}\right)$ and each $\theta_{j} \in \Theta_{j}\left(\theta_{i}\right)$ there is some $t_{j} \in T_{j}\left(t_{i}\right)$ such that $\theta_{j} \in \Theta_{j}\left(t_{i}\right)$, and, by inductive assumption, each $\theta_{j} \in \Theta_{j}\left(\theta_{i}\right)$ expresses $n$-fold full belief in caution. Therefore, each $\theta_{i} \in \Theta_{i}\left(t_{i}\right)$ expresses $(n+1)$-fold full belief in caution.

Proof of Lemma 4,2. We show this statement by induction. First we show that if $t_{i}$ primarily believes in $j$ 's rationality, then each $\theta_{i} \in \Theta_{i}\left(t_{i}\right)$ primarily believes in utilities nearest to $u$. Let $\left(c_{j}, \theta_{j}\right)$ be a pair deemed possible in the level-1 belief of $\theta_{i}$. Consider its correspondence $\left(c_{j}, t_{j}\right)$ in level-1 belief of $t_{i}$. Since $t_{i}$ primarily believes in $j$ 's rationality, $c_{j}$ is rational for $t_{j}$. It follows that $c_{j} \in C_{j 1} \in \Pi_{j}\left(t_{j}\right)$. By Lemma 21 and construction, it follows that $w_{j}\left(\theta_{j}\right)=u_{j}$. Since $u_{j}$ is the nearest function to itself among all utility functions in $V_{j}$, we have shown that $\theta_{i}$ primarily believes in utilities nearest to $u$.

Suppose we have shown that, for each $i \in I$, if $t_{i}$ expresses $n$-fold full belief in primary belief in rationality then each $\theta_{i} \in \Theta_{i}\left(t_{i}\right)$ expresses $n$-fold full belief in primary belief in utilities nearest to $u$. Now suppose that $t_{i}$ expresses $(n+1)$-fold full belief in primary belief in rationality, i.e., each $t_{j} \in T_{j}\left(t_{i}\right)$ expresses $n$-fold full belief in primary belief in rationality. Since, by construction, for each $\theta_{i} \in \Theta_{i}\left(t_{i}\right)$ and each $\theta_{j} \in \Theta_{j}\left(\theta_{i}\right)$ there is some $t_{j} \in T_{j}\left(t_{i}\right)$ such that $\theta_{j} \in \Theta_{j}\left(t_{j}\right)$, it follows that, by inductive assumption, each $\theta_{j} \in \Theta_{j}\left(\theta_{i}\right)$ expresses $n$-fold full belief in primary belief in utilities nearest to $u$. Therefore, each $\theta_{i} \in \Theta_{i}\left(t_{i}\right)$ expresses $(n+1)$-fold full belief in primary belief in utilities nearest to $u$.

Proof of Lemma 4,3. We show this statement by induction. First we show that if $\theta_{i}$ is cautious, then $t_{i}\left(E_{i}\left(\theta_{i}\right)\right)$ is also cautious. Let $c_{j} \in C_{j}$ and $t_{j} \in T_{j}\left(t_{i}\left(E_{i}\left(\theta_{i}\right)\right)\right)$. By construction, $t_{j}=t_{j}\left(E_{j}\right)$ for some $E_{j} \in \mathbb{E}_{j}$, and there is some $\theta_{j} \in E_{j}$ which is deemed possible by $\theta_{i}$. Since $\theta_{i}$ is cautious, there is some $\theta_{j}^{\prime}$ with $\beta_{j}\left(\theta_{j}^{\prime}\right)=\beta_{j}\left(\theta_{j}\right)$, i.e., $\theta_{j}^{\prime} \in E_{j}$, such that $\left(c_{j}, \theta_{j}^{\prime}\right)$ is deemed possible by $\theta_{i}$. By construction, $\left(c_{j}, t_{j}\right)$ is deemed possible by $t_{i}\left(E_{i}\left(\theta_{i}\right)\right)$.

Suppose we have shown that, for each $i \in I$, if $\theta_{i}$ expresses $n$-fold full belief in caution then so does $t_{i}\left(E_{i}\left(\theta_{i}\right)\right)$. Now suppose that $\theta_{i}$ expresses $(n+1)$-fold full be- 
lief in caution, i.e., each $\theta_{j} \in \Theta_{j}\left(\theta_{i}\right)$ expresses $n$-fold full belief in caution. Since, by construction, for each $t_{j} \in T_{j}\left(t_{i}\left(E_{i}\left(\theta_{i}\right)\right)\right)$, there is some $\theta_{j} \in \Theta_{j}\left(\theta_{i}\right)$ such that $t_{j}=t_{j}\left(E_{j}\left(\theta_{j}\right)\right)$, by inductive assumption $t_{j}$ expresses $n$-fold full belief in caution. Therefore, $t_{i}\left(E_{i}\left(\theta_{i}\right)\right)$ expresses $(n+1)$-fold full belief in caution.

Proof of Lemma 4, 4. We show this statement by induction. First we show that if $\theta_{i}$ is cautious, primarily believes in utilities nearest to $u$, and believes in that a best choice is supported by utilities nearest to $u$, then $t_{i}\left(E_{i}\left(\theta_{i}\right)\right)$ primarily believes in $j$ 's rationality. Let $\left(c_{j}, t_{j}\right)$ be a choice-type pair which is deemed possible in $t_{i}\left(E_{i}\left(\theta_{i}\right)\right)$ 's level-1 belief. By construction $t_{j}=t_{j}\left(E_{j}\right)$ for some $E_{j} \in \mathbb{E}_{j}$, and for some $\theta_{j} \in E_{j},\left(c_{j}, \theta_{j}\right)$ is deemed possible in $\theta_{i}$ 's level-1 belief. Since $\theta_{i}$ primarily believes in utilities nearest to $u$, it follows that

$$
d\left(w_{j}\left(\theta_{j}\right), u_{j}\right) \leq d\left(w_{j}\left(\theta_{j}^{\prime}\right), u_{j}\right) \text { for all } \theta_{j}^{\prime} \in E_{j} .
$$

Suppose that $c_{j}$ is not optimal for $t_{j}$. Let $c_{j}^{\prime}$ be a choice optimal to $t_{j}$. Since $\theta_{i}$ is cautious, there is some $\theta_{j}^{v_{j}} \in E_{j}$ such that $\left(c_{j}, \theta_{j}^{v_{j}}\right)$ is deemed possible by $\theta_{i}$. Then since $\theta_{i}$ believes in that a best choice is supported by utilities nearest to $u$, it follows that $d\left(v_{j}, u_{j}\right)=d\left(w_{j}\left(\theta_{j}^{v_{j}}\right), u_{j}\right)<d\left(w_{j}\left(\theta_{j}\right), u_{j}\right)$, which is contradictory to (1). Therefore $c_{j}$ is optimal for $t_{j}$. Here we have shown that $t_{i}\left(E_{i}\left(\theta_{i}\right)\right)$ primarily believes in $j$ 's rationality.

Suppose we have shown that, for each $i \in I$, if $\theta_{i}$ expresses $n$-fold full belief in caution, primary belief in utilities nearest to $u$, and that a best choice is supported by utilities nearest to $u$, then $t_{i}\left(E_{i}\left(\theta_{i}\right)\right)$ expresses $n$-fold belief in primary belief in rationality. Now suppose that $\theta_{i}$ expresses $(n+1)$-fold full belief in caution, primary belief in utilities nearest to $u$, and that a best choice is supported by utilities nearest to $u$, i.e., each $\theta_{j} \in \Theta_{j}\left(\theta_{i}\right)$ expresses $n$-fold full belief in caution, primary belief in utilities nearest to $u$, and that a best choice is supported by utilities nearest to $u$. Since, by construction, for each $t_{j} \in T_{j}\left(t_{i}\left(E_{i}\left(\theta_{i}\right)\right)\right)$, there is some $\theta_{j} \in \Theta_{j}\left(\theta_{i}\right)$ such that $t_{j}=t_{j}\left(E_{j}\left(\theta_{j}\right)\right)$, by inductive assumption $t_{j}$ expresses $n$-fold full belief in primary belief in rationality. Therefore, $t_{i}\left(E_{i}\left(\theta_{i}\right)\right)$ expresses $(n+1)$-fold full belief in primary belief in rationality.

Proof of Lemma 4,5. We show this statement by induction. First we show that if $t_{i}$ is caution and respects $j$ 's preferences, then each $\theta_{i} \in \Theta_{i}\left(t_{i}\right)$ expresses $u$-centered belief. It can be seen that if $t_{i}$ is cautious and respects $j$ 's preferences, then we can combine all types deemed possible by $t_{i}$ with the same belief into one type without hurting the caution and respect of $j$ 's preference, and every choice optimal for $t_{i}$ is still optimal for this new type and vice versa. Therefore, without loss of generality we can assume that for each $t_{j}, t_{j}^{\prime} \in T_{j}, b_{j}\left(t_{j}\right) \neq b_{j}\left(t_{j}^{\prime}\right)$. Let $c_{j}, c_{j}^{\prime} \in C_{j}, \theta_{j} \in \Theta_{j}$, and $v_{j}, v_{j}^{\prime} \in V_{j}$ such that $\left(c_{j}, \theta_{j}^{v_{j}}\right)$ and $\left(c_{j}^{\prime}, \theta_{j}^{v_{j}^{\prime}}\right)$ are deemed possible by $\theta_{i}$ with $d\left(v_{j}, u_{j}\right)<d\left(v_{j}^{\prime}, u_{j}\right)$. Since each type in $T_{i}$ has a distinct lexicographic belief, it follows that $\theta_{j}^{v_{j}}, \theta_{j}^{v_{j}^{\prime}} \in$ $\Theta_{j}\left(t_{j}\right)$ for some $t_{j} \in T_{j}$. By construction it follows that (1) $t_{i}$ deems both $\left(c_{j}, t_{j}\right)$ and $\left(c_{j}^{\prime}, t_{j}\right)$ possible, and (2) $u_{j}\left(c_{j}, t_{i}\right)>u_{j}\left(c_{j}^{\prime}, t_{i}\right)$. Since $t_{i}$ respects $j$ 's preferences, $t_{i}$ deems $\left(c_{j}, t_{j}\right)$ infinitely more likely than $\left(c_{j}^{\prime}, t_{j}\right)$, which corresponds to that $\theta_{i}$ deems $\left(c_{j}, \theta_{j}^{v_{j}}\right)$ infinitely more likely than $\left(c_{j}^{\prime}, \theta_{j}^{v_{j}^{\prime}}\right)$. Here we have shown that $\theta_{i}$ expresses $u$-centered belief. 
Suppose we have shown that, for each $i \in I$, if $t_{i}$ expresses $n$-fold full belief in respect of preferences then each $\theta_{i} \in \Theta_{i}\left(t_{i}\right)$ expresses $n$-fold full belief in $u$-centered belief. Now suppose that $t_{i}$ expresses $(n+1)$-fold full belief in respect of preferences, i.e., each $t_{j} \in T_{j}\left(t_{i}\right)$ expresses $n$-fold full belief respect of preferences. Since, by construction, for each $\theta_{i} \in \Theta_{i}\left(t_{i}\right)$ and each $\theta_{j} \in \Theta_{j}\left(\theta_{i}\right)$ there is some $t_{j} \in T_{j}\left(t_{i}\right)$ such that $\theta_{j} \in \Theta_{j}\left(t_{j}\right)$, by inductive assumption it follows that each $\theta_{j} \in \Theta_{j}\left(\theta_{i}\right)$ expresses $n$-fold full belief in $u$-centered belief. Therefore, each $\theta_{i} \in \Theta_{i}\left(t_{i}\right)$ expresses $(n+1)$-fold full belief in $u$-centered belief.

Proof of Lemma 4]6. We show this statement by induction. First we show that if $\theta_{i}$ is cautious, has a $u$-centered belief, and believes that a better choice is supported by utilities nearer to $u$, then $t_{i}\left(E_{i}\left(\theta_{i}\right)\right)$ respects $j$ 's preferences. First, since $\theta_{i}$ is cautious, By Lemma 4 $3, t_{i}\left(E_{i}\left(\theta_{i}\right)\right)$ is also cautious. Let $c_{j}, c_{j}^{\prime} \in C_{j}$ and $t_{j} \in T_{j}\left(t_{i}\left(E_{i}\left(\theta_{i}\right)\right)\right)$ with $t_{j}$ prefers $c_{j}$ to $c_{j}^{\prime}$. By construction $t_{j}=t_{j}\left(E_{j}\right)$ for some $E_{j} \in \mathbb{E}_{j}$, and, since $\theta_{i}$ is cautious, there are $\theta_{j}, \theta_{j}^{\prime} \in E_{j}$ such that $\theta_{i}$ deems $\left(c_{j}, \theta_{j}\right)$ and $\left(c_{j}^{\prime}, \theta_{j}^{\prime}\right)$ possible. Since $\beta_{j}\left(\theta_{j}\right)=\beta_{j}\left(\theta_{j}^{\prime}\right)$ and $\theta_{j}$ has the same probability distribution over $C_{i}$ at each level as $t_{j}$, it follows that $u_{j}\left(c_{j}, \theta_{j}\right)>u_{j}\left(c_{j}^{\prime}, \theta_{j}\right)$. Since $\theta_{i}$ believes that a better choice is supported by utilities nearer to $u$, it follows that $d\left(w_{j}\left(\theta_{j}\right), u_{j}\right)<d\left(w_{j}\left(\theta_{j}^{\prime}\right), u_{j}\right)$. Since $\theta_{i}$ has a $u$-centered belief, it follows that $\theta_{i}$ deems $\left(c_{j}, \theta_{j}\right)$ infinitely more likely than $\left(c_{j}^{\prime}, \theta_{j}^{\prime}\right)$, which implies that $t_{i}\left(E_{i}\left(\theta_{i}\right)\right)$ deems $\left(c_{j}, t_{j}\right)$ infinitely more likely than $\left(c_{j}^{\prime}, t_{j}\right)$. Therefore, $t_{i}\left(E_{i}\left(\theta_{i}\right)\right)$ respects $j$ 's preferences.

Suppose we have shown that, for each $i \in I$, if $\theta_{i}$ expresses $n$-fold full belief in caution, $u$-centered belief, and that a better choice is supported by utilities nearer to $u$, then $t_{i}\left(E_{i}\left(\theta_{i}\right)\right)$ expresses $n$-fold full belief in respect of preferences. Now suppose that $\theta_{i}$ expresses $(n+1)$-fold full belief in caution, $u$-centered belief, and that a better choice is supported by utilities nearer to $u$, i.e., each $\theta_{j} \in \Theta_{j}\left(\theta_{i}\right)$ expresses $n$-fold full belief in caution, $u$-centered belief, and that a better choice is supported by utilities nearer to $u$. Since, by construction, for each $t_{j} \in T_{j}\left(t_{i}\left(E_{i}\left(\theta_{i}\right)\right)\right)$, there is some $\theta_{j} \in \Theta_{j}\left(\theta_{i}\right)$ such that $t_{j}=t_{j}\left(E_{j}\left(\theta_{j}\right)\right)$, by inductive assumption $t_{j}$ expresses $n$-fold full belief in respect of preferences. Therefore, $t_{i}\left(E_{i}\left(\theta_{i}\right)\right)$ expresses $(n+1)$-fold full belief in respect of preferences.

Proof of Lemma 4,7. We show this statement by induction. First we show that if $t_{i}$ primarily believes in $j$ 's rationality, then each $\theta_{i} \in \Theta_{i}\left(t_{i}\right)$ primarily believes in $u$. Let $\left(c_{j}, \theta_{j}\right)$ be a pair deemed possible in the level-1 belief of $\theta_{i}$. Consider its corresponding $\left(c_{j}, t_{j}\right)$ in level-1 belief of $t_{i}$. Since $t_{i}$ primarily believes in $j$ 's rationality, $c_{j}$ is rational for $t_{j}$. It follows that $c_{j} \in C_{j 1} \in \Pi_{j}\left(t_{j}\right)$. By construction, it follows that $w_{j}\left(\theta_{j}\right)=u_{j}$. Here we have shown that $\theta_{i}$ primarily believes in $u$.

Suppose we have shown that, for each $i \in I$, if $t_{i}$ expresses $n$-fold full belief in primary belief in rationality then each $\theta_{i} \in \Theta_{i}\left(t_{i}\right)$ expresses $n$-fold full belief in primary belief in $u$. Now suppose that $t_{i}$ expresses $(n+1)$-fold full belief in primary belief in rationality, i.e., each $t_{j} \in T_{j}\left(t_{i}\right)$ expresses $n$-fold full belief in primary belief in rationality. Since, by construction, for each $\theta_{i} \in \Theta_{i}\left(t_{i}\right)$ and each $\theta_{j} \in \Theta_{j}\left(\theta_{i}\right)$ there is some $t_{j} \in T_{j}\left(t_{i}\right)$ such that $\theta_{j} \in \Theta_{j}\left(t_{j}\right)$, it follows that, by inductive assumption, each $\theta_{j} \in \Theta_{j}\left(\theta_{i}\right)$ expresses $n$-fold full belief in primary belief in rationality. Therefore, each $\theta_{i} \in \Theta_{i}\left(t_{i}\right)$ expresses $(n+1)$-fold full belief in primary belief in $u$.

Proof of Lemma 4,8. We show this statement by induction. First we show that if $\theta_{i}$ 
believes in $j$ 's rationality and primarily believes in $u$, then $t_{i}\left(E_{i}\left(\theta_{i}\right)\right)$ primarily believes in $j$ 's rationality. Let $\left(c_{j}, t_{j}\right)$ be a choice-type pair which is deemed possible in $t_{i}\left(E_{i}\left(\theta_{i}\right)\right)$ 's level-1 belief. By construction $t_{j}=t_{j}\left(E_{j}\right)$ for some $E_{j} \in \mathbb{E}_{j}$, and for some $\theta_{j} \in E_{j},\left(c_{j}, \theta_{j}\right)$ is deemed possible in $\theta_{i}$ 's level-1 belief. Since $\theta_{i}$ primarily believes in $u$, it follows that $w_{j}\left(\theta_{j}\right)=u_{j}$. Also, since $\theta_{i}$ believes $j$ 's rationality, it follows that $c_{j}$ is rational at $u_{j}$ under $\beta_{j}\left(\theta_{j}\right)$, i.e., $b_{i}\left(t_{j}\right)$. Therefore $c_{j}$ is rational for $t_{j}$. Here we have shown that $t_{i}\left(E_{i}\left(\theta_{i}\right)\right)$ primarily believes in $j$ 's rationality.

Suppose we have shown that, for each $i \in I$, if $\theta_{i}$ expresses $n$-fold full belief in rationality and primary belief in $u$, then $t_{i}\left(E_{i}\left(\theta_{i}\right)\right)$ expresses $n$-fold belief in primary belief in rationality. Now suppose that $\theta_{i}$ expresses $(n+1)$-fold full belief in rationality and primary belief in $u$, i.e., each $\theta_{j} \in \Theta_{j}\left(\theta_{i}\right)$ expresses $n$-fold full belief in rationality and primary belief in $u$. Since, by construction, for each $t_{j} \in T_{j}\left(t_{i}\left(E_{i}\left(\theta_{i}\right)\right)\right)$, there is some $\theta_{j} \in \Theta_{j}\left(\theta_{i}\right)$ such that $t_{j}=t_{j}\left(E_{j}\left(\theta_{j}\right)\right)$, by inductive assumption $t_{j}$ expresses $n$ fold full belief in primary belief in rationality. Therefore, $t_{i}\left(E_{i}\left(\theta_{i}\right)\right)$ expresses $(n+1)$ fold full belief in primary belief in rationality.

Proof of Lemma 49. We show this statement by induction. Let $\theta_{i} \in \Theta_{i}\left(t_{i}\right)$. First we show that if $t_{i}$ assumes in $j$ 's rationality, $\theta_{i}$ prior assumes $u$ and assumes that every good choice is supported. Let $c_{j} \in C_{j}$ be optimal for some cautious type of $j$ whose assigned utility function is $u_{j}$ within an epistemic model with incomplete information. It is easy to see that $c_{j}$ is optimal for its corresponding type, which is also cautious by Lemma 42 , in any complete information model constructed from the one with incomplete information. Since $t_{i}$ assumes $j$ 's rationality, $t_{i}$ deems possible a cautious type $t_{j}$ for which $c_{j}$ is optimal. By construction, some $\theta_{j} \in \Theta_{j}\left(t_{j}\right)$ is deemed possible by $\theta_{i}$. Since $t_{i}$ is cautious, $\left(c_{j}, t_{j}\right)$ is deemed possible by $t_{i}$, and, by construction $\left(c_{j}, \theta_{j 1}\left(t_{j}\right)\right)$ is deemed possible by $\theta_{i}$. Since $w_{j}\left(\theta_{j 1}\left(t_{j}\right)\right)=u_{j}$ and $c_{j}$ is optimal for $\theta_{j 1}\left(t_{j}\right)$, it follows that $\theta_{i}$ assumes that every good choice is supported.

Let $\left(c_{j}, \theta_{j}\right)$ with $\theta_{j}$ cautious deemed possible by $\theta_{i}$ satisfying $w_{j}\left(\theta_{j}\right)=u_{j}$ and $\left(c_{j}^{\prime}, \theta_{j}^{\prime}\right)$ a pair which does not satisfy that condition. Let $\left(c_{j}, t_{j}\right)$ and $\left(c_{j}^{\prime}, t_{j}^{\prime}\right)$ be the pairs occurring in the belief of $t_{i}$ corresponding to $\left(c_{j}, \theta_{j}\right)$ and $\left(c_{j}^{\prime}, \theta_{j}^{\prime}\right)$. Since $c_{j}$ is rational to $\theta_{j}$ and $w_{j}\left(\theta_{j}\right)=u_{j}$, it follows that $c_{j}$ is optimal for $t_{j}$. On the other hand, $c_{j}^{\prime}$ is not optimal for $t_{j}^{\prime}$. Since $t_{i}$ assumes $j$ 's rationality, $t_{i}$ deems $\left(c_{j}, t_{j}\right)$ infinitely more likely than $\left(c_{j}^{\prime}, t_{j}^{\prime}\right)$. By construction, $\theta_{i}$ deems $\left(c_{j}, \theta_{j}\right)$ infinitely more likely than $\left(c_{j}^{\prime}, \theta_{j}^{\prime}\right)$. Here we have shown that $\theta_{i}$ prior assumes $u$.

Now we show the other direction: suppose that if $\theta_{i} \in \Theta_{i}$ prior assumes $u$ and assumes that every good choice is supported, we prove that $t_{i}\left(E_{i}\left(\theta_{i}\right)\right)$ assumes $j$ 's rationality. Suppose that $c_{j}$ is optimal for some cautious type within some epistemic model with complete information. It can be seen by construction that $c_{j}$ is optimal for some cautious type with $u_{i}$ as its assigned utility function within some epistemic model with incomplete information which corresponds to that complete information model. Since $\theta_{i}$ believes in that every good choice is supported, $\theta_{i}$ deems possible a cautious type $\theta_{j}$ such that $w_{j}\left(\theta_{j}\right)=u_{j}$ and $c_{j}$ is optimal for $\theta_{j}$. By construction it follows that $t_{i}\left(E_{i}\left(\theta_{i}\right)\right)$ deems $t_{j}\left(E_{j}\left(\theta_{j}\right)\right)$ possible for which $c_{j}$ is optimal.

Let $\left(c_{j}, t_{j}\right)$ with $t_{j}$ cautious be a pair which is deemed possible by $t_{i}\left(E_{i}\left(\theta_{i}\right)\right)$ satisfying that $c_{j}$ is optimal for $t_{j}$, and $\left(c_{j}^{\prime}, t_{j}^{\prime}\right)$ be a pair deemed possible by $t_{i}\left(E_{i}\left(\theta_{i}\right)\right)$ which does not satisfy that condition. Since $\theta_{i}$ assumes that every good choice is 
supported, there is some $\theta_{j} \in \Theta_{j}\left(\theta_{i}\right)$ corresponding to $t_{j}$ with $w_{j}\left(\theta_{j}\right)=u_{j}$ such that $\left(c_{j}, \theta_{j}\right)$ is deemed possible by $\theta_{i}$. On the other hand, since $\theta_{i}$ believes in rationality, for each $\theta_{j}^{\prime}$ such that $\left(c_{j}^{\prime}, \theta_{j}^{\prime}\right)$ is deemed possible by $\theta_{i}$, it holds that $w_{j}\left(\theta_{j}^{\prime}\right) \neq u_{j}$. Since $\theta_{i}$ prior assumes $u, \theta_{i}$ deems $\left(c_{j}, \theta_{j}\right)$ infinitely more likely than $\left(c_{j}^{\prime}, \theta_{j}^{\prime}\right)$. It follows that $t_{i}\left(E_{i}\left(\theta_{i}\right)\right)$ deems $\left(c_{j}, t_{j}\right)$ infinitely more likely than $\left(c_{j}^{\prime}, t_{j}^{\prime}\right)$. Here we have shown that $t_{i}\left(E_{i}\left(\theta_{i}\right)\right)$ assumes $j$ 's rationality.

Suppose that, for some $n \in \mathbb{N}$, we have shown that for each $k \leq n$,

(n1) if $t_{i} \in T_{i}$ expresses $k$-fold assumption of rationality, then each $\theta_{i} \in \Theta_{i}\left(t_{i}\right)$ expresses $k$-fold assumption of prior $u$ and that every good choice is supported;

(n2) If $\theta_{i} \in \Theta_{i}$ expresses $k$-fold full belief of rationality and $k$-fold assumption of prior $u$ and that every good choice is supported, then $t_{i}\left(E_{i}\left(\theta_{i}\right)\right)$ expresses $k$-fold assumption of rationality.

Now we show that these two statements hold for $n+1$. First, suppose that $t_{i} \in T_{i}$ expresses $(n+1)$-fold assumption of rationality. Let $c_{j} \in C_{j}$ be a choice of $j$ optimal for some cautious type whose assigned utility function is $u_{j}$ that expresses up to $n$-fold assumption of prior $u$ and that every good choice is supported. Then it is easy to see that (1) by inductive assumption, in the constructed complete information model the corresponding type expresses $n$-fold assumption of rationality, and (2) $c_{j}$ is optimal for that type. Since $t_{i}$ expresses $(n+1)$-fold assumption of rationality, $t_{i}$ deems possible a cautious type $t_{j}$ that expresses up to $n$-fold assumption of rationality and for which $c_{j}$ is optimal. By construction, it follows that $\theta_{i}$ deems possible some $\theta_{j} \in \Theta_{j}\left(t_{j}\right)$. By inductive assumption it follows that each $\theta_{j} \in \Theta_{j}\left(t_{j}\right)$ expresses $n$-fold assumption of in that every good choice is supported. Since $\theta_{i}$ expresses common belief in caution and rationality it follows that $\theta_{i}$ deems $\left(c_{j}, \theta_{j 1}\right)$ for $\theta_{j 1} \in \Theta_{j}\left(t_{j}\right)$ (that is, $\left.w_{j}\left(\theta_{j 1}\right)=u_{j}\right)$.

Let $\left(c_{j}, \theta_{j}\right)$ with $\theta_{j}$ cautious deemed possible by $\theta_{i}$ satisfying that $\theta_{j}$ expresses up to $n$-fold assumption of prior $u$ and that every good choice is supported and $w_{j}\left(\theta_{j}\right)=u_{j}$ and $\left(c_{j}^{\prime}, \theta_{j}^{\prime}\right)$ a pair which does not satisfy those conditions. Let $\left(c_{j}, t_{j}\right)$ and $\left(c_{j}^{\prime}, t_{j}^{\prime}\right)$ be the pairs occurring in the belief of $t_{i}$ corresponding to $\left(c_{j}, \theta_{j}\right)$ and $\left(c_{j}^{\prime}, \theta_{j}^{\prime}\right)$. Since $c_{j}$ is rational $\theta_{j}$ with $w_{j}\left(\theta_{j}\right)=u_{j}$, it follows that $c_{j}$ is optimal for $t_{j}$. Also, by inductive assumption, it follows that $t_{j}$ expresses up to $n$-fold assumption of rationality. On the other hand, it can be seen that $\left(c_{j}^{\prime}, t_{j}^{\prime}\right)$ does not satisfy these conditions. Since $t_{i}$ expresses $(n+1)$-fold of assumptions of rationality, $t_{i}$ deems $\left(c_{j}, t_{j}\right)$ infinitely more likely than $\left(c_{j}^{\prime}, t_{j}^{\prime}\right)$. By construction, $\theta_{i}$ deems $\left(c_{j}, \theta_{j}\right)$ infinitely more likely than $\left(c_{j}^{\prime}, \theta_{j}^{\prime}\right)$. Here we have shown that $\theta_{i}$ expresses $(n+1)$-fold assumption of prior $u$ and that every good choice is supported.

Now suppose that $\theta_{i} \in \Theta_{i}$ expresses $(n+1)$-fold assumption of prior $u$ and that every good choice is supported. Let $c_{j} \in C_{j}$ be a choice of $j$ optimal for some cautious type that expresses to $n$-fold assumption of rationality. By inductive assumption it follows that the corresponding type within some incomplete information model also expresses $n$-fold assumption of prior $u$ and that every good choice is supported. It can be seen that $c_{j}$ is optimal to the constructed type having $u_{j}$ as its utility function and the type expresses up to $n$-fold assumption of prior $u$ and that every good choice is supported. Then $\theta_{i}$ deems possible a type $\theta_{j}$ with $w_{j}\left(\theta_{j}\right)=u_{j}$ for player $j$ which expresses up to $n$-fold assumption of prior $u$ and that every good choice is supported 
for which $c_{j}$ is optimal. By inductive assumption it follows that $t_{i}\left(E_{i}\left(\theta_{i}\right)\right)$ deems possible $t_{j}\left(E_{j}\left(\theta_{j}\right)\right)$ which expresses $n$-fold assumption of rationality and for which $c_{j}$ is optimal.

Let $\left(c_{j}, t_{j}\right)$ be a pair with $t_{j}$ cautious deemed possible by $t_{i}\left(E_{i}\left(\theta_{i}\right)\right)$ where $t_{j}$ expresses up to $n$-fold assumption of rationality and $c_{j}$ is optimal for $t_{j}$, and let $\left(c_{j}^{\prime}, t_{j}^{\prime}\right)$ be a pair that does not satisfy this property. It can be seen that there is some $\theta_{j}$ corresponding to $t_{j}$ such that $\theta_{j}$ is cautious and expresses up to $n$-fold assumption of prior $u$ and that every good choice is supported and $w_{j}\left(\theta_{j}\right)=u_{j}$, while $\left(c_{j}^{\prime}, \theta_{j}^{\prime}\right)$ does not satisfy this property for any $\theta_{j}^{\prime}$ deemed possible by $\theta_{i}$ since $\theta_{i}$ expresses $n$-fold full belief of rationality. Therefore $t_{i}\left(E_{i}\left(\theta_{i}\right)\right)$ deems $\left(c_{j}, t_{j}\right)$ infinitely more likely than $\left(c_{j}^{\prime}, t_{j}^{\prime}\right)$. Here we have shown that $t_{i}\left(E_{i}\left(\theta_{i}\right)\right)$ expresses $(n+1)$-fold assumption of rationality.

Proof of Lemma 5, 1. The only-if part holds automatically. To show the if part, we need first to show that each weak cautious type can be extended into a cautious one without changing the set of choices rational for it. It is done by an interpolation method as follows. Let $t_{i}$ be a type satisfying weak caution with $b_{i}\left(t_{i}\right)=\left(b_{i 1}, \ldots, b_{i K}\right)$, $c_{j} \in C_{j}$, and $t_{j} \in T_{j}\left(t_{i}\right)$. Suppose that $\left(c_{j}, t_{j}\right)$ is not deemed possible by $t_{i}$. Since $t_{i}$ is weakly cautious, there is some $t_{j}^{\prime} \in T_{j}$ such that for some $k \in\{1, \ldots, K\}, b_{i k}\left(c_{j}, t_{j}^{\prime}\right)>0$. Now we extend $\left(b_{i 1}, \ldots, b_{i K}\right)$ into $\left(b_{i 1}^{\prime}, \ldots, b_{i, K+1}^{\prime}\right)$ by letting (1) $b_{i t}^{\prime}=b_{i t}$ for each $t \leq k$, (2) $b_{i t}^{\prime}=b_{i, t-1}$ for each $t>k+1$, and (3) $b_{i, k+1}^{\prime}$ is obtained by replacing every occurrence of $\left(c_{j}, t_{j}^{\prime}\right)$ by $\left(c_{j}, t_{j}\right)$ in the distribution of $b_{i k}$. We call $b_{i, k+1}^{\prime}$ a doppelganger of $b_{i k}$. It can be seen that for each $c_{i} \in C_{i}$, and a doppelganger $b_{i, k+1}^{\prime}$ of $b_{i k}$, $u_{i}\left(c_{i}, b_{i, k+1}^{\prime}\right)=u_{i}\left(c_{i}, b_{i k}\right)$. By repeatedly interpolating doppelgangers into $b_{i}\left(t_{i}\right)$ for each missed choice-type pairs, finally we obtain a lexicographic belief $\left(b_{i 1}^{\prime}, \ldots, b_{i K^{\prime}}^{\prime}\right)$ that satisfies caution. We use $\bar{t}_{i}$ to denote the type with belief $\left(b_{i 1}^{\prime}, \ldots, b_{i K^{\prime}}^{\prime}\right) \cdot \bar{t}_{i}$ is called a cautious extension of $t_{i}$. We have the following lemma.

Lemma 61 (Extended type preserves rational choices). Let $t_{i}$ be a weakly cautious type and $\bar{t}_{i}$ a cautious extension of $t_{i}$. Then $c_{i} \in C_{i}$ is rational for $t_{i}$ if and only if it is rational for $\bar{t}_{i}$.

Proof. (Only-if) Suppose that $c_{i}$ is not rational for $\bar{t}_{i}$. Then there is some $c_{i}^{\prime} \in C_{i}$ which is preferred $c_{i}$ under $b_{i}\left(\bar{t}_{i}\right)=\left(b_{i 1}^{\prime}, \ldots, b_{i K^{\prime}}^{\prime}\right)$, that is, there is some $k^{\prime} \in\left\{0, \ldots, K^{\prime}\right\}$ such that $u_{i}\left(c_{i}, b_{i \ell}^{\prime}\right)=u_{i}\left(c_{i}^{\prime}, b_{i \ell}^{\prime}\right)$ for each $\ell \leq k^{\prime}$ and $u_{i}\left(c_{i}, b_{i, k^{\prime}+1}\right)<u_{i}\left(c_{i}^{\prime}, b_{i, k^{\prime}+1}\right)$. Let $b_{i, k+1}$ be the entry in $b_{i}\left(t_{i}\right)$ such that $b_{i, k^{\prime}+1}^{\prime}$ is its doppelganger. It follows that in the original $b_{i}\left(t_{i}\right)=\left(b_{i 1}, \ldots, b_{i K}\right), u_{i}\left(c_{i}, b_{i \ell}\right)=u_{i}\left(c_{i}^{\prime}, b_{i \ell}\right)$ for each $\ell \leq k$ and $u_{i}\left(c_{i}, b_{i, k+1}\right)<$ $u_{i}\left(c_{i}^{\prime}, b_{i, k+1}\right)$. Hence $c_{i}$ is not rational for $t_{i}$.

(If) Suppose that $c_{i}$ is not rational for $t_{i}$. Then there is some $c_{i}^{\prime} \in C_{i}$ which is preferred $c_{i}$ under $b_{i}\left(t_{i}\right)=\left(b_{i 1}, \ldots, b_{i K}\right)$, that is, there is some $k \in\{0, \ldots, K\}$ such that $u_{i}\left(c_{i}, b_{i \ell}\right)=u_{i}\left(c_{i}^{\prime}, b_{i \ell}\right)$ for each $\ell \leq k$ and $u_{i}\left(c_{i}, b_{i, k+1}\right)<u_{i}\left(c_{i}^{\prime}, b_{i, k+1}\right)$. Let $b_{i, k^{\prime}+1}^{\prime}$ be the corresponding doppelganger in $b_{i}\left(\bar{t}_{i}\right)$ to $b_{i, k+1}$. It follows that in the original $u_{i}\left(c_{i}, b_{i \ell}^{\prime}\right)=u_{i}\left(c_{i}^{\prime}, b_{i \ell}^{\prime}\right)$ for each $\ell \leq k^{\prime}$ and $u_{i}\left(c_{i}, b_{i, k^{\prime}+1}^{\prime}\right)<u_{i}\left(c_{i}^{\prime}, b_{i, k^{\prime}+1}^{\prime}\right)$. Hence $c_{i}$ is not rational for $\bar{t}_{i}$.

Proof of Lemma 5,1 (Continued) Since caution implies weak caution, the only-if part holds automatically. For the if part, suppose that $c_{i}^{*}$ is rational for some $t_{i}^{*} \in T_{i}$ which expresses common full belief in weak caution and primary belief in rational- 
ity. Consider an epistemic model $\left(\bar{T}_{i}, \bar{b}_{i}\right)_{i \in I}$ such that for each $i \in I, \bar{T}_{i}=\left\{\bar{t}_{i}: t_{i} \in T_{i}\right\}$ and $\bar{b}_{i}\left(\bar{t}_{i}\right)$ is a cautious extension of $b_{i}\left(t_{i}\right)$ with replacing each occurrence of $t_{j}$ by $\bar{t}_{j}$. By Lemma 6 1, since $c_{i}^{*}$ is rational for $t_{i}^{*}$, it is also rational for $\overline{t_{i}^{*}}$. Also, it can be seen by construction that $\overline{t_{i}^{*}}$ expresses common full belief in caution. Also, since the interpolation always put doppelgangers after the original one, it does not change the level-1 belief, and consequently $\overline{t_{i}^{*}}$ expresses common full belief in primary belief in rationality. Therefore, $c_{i}^{*}$ is permissible.

\section{References}

1. Asheim GB (2001) Proper rationalizability in lexicographic beliefs. Int J Game Theory 30:453-478.

2. Battigalli P (2003) Rationalizability in infinite, dynamic games of incomplete information. Res Econ 57:1-38.

3. Battigalli P, Siniscalchi M (2003) Rationalization and incomplete information. BE J Theor Econ 61:165-184.

4. Battigalli P, Siniscalchi M (2007) Interactive epistemology in games with payoff uncertainty. Res Econ 3:1534-5963.

5. Bernheim D (1984) Rationalizable strategic behavior. Econometrica 52:1007-1028

6. Blume L, Brandenburger A, Dekel E (1991a) Lexicographic probabilities and choice under uncertainty. Econometrica 59:61-79.

7. Blume L, Brandenburger A, Dekel E (1991b) Lexicographic probabilities and equilibrium refinements. Econometrica 59:81-98.

8. Böge W, Eisele T (1979) On solutions of Bayesian games. Int J Game Theory 8:193-215.

9. Börgers T (1994) Weak dominance and approximate common knowledge. J Econ Theory 64:265-276.

10. Börgers T, Samuelson L (1992) "Cautious" utility maximization and iterated weak dominance. Int J Game Theory 21:13-25.

11. Brandenburger A (1992) Lexicographic probabilities and iterated admissibility. In Dasgupta J et al. (ed) Economic Analysis of Markets and Games. MIT Press, Cambridge, pp 282-290.

12. Brandenburger A, Friedenberge A, Keisler J (2008) Admissibility in games. Econometrica 76:307352.

13. Dekel E, Fudenberg D (1990) Rational behavior with payoff uncertainty. J Econ Theory 52:243-267.

14. Dekel E, Siniscalchi M (2015) Epistemic game theory. In Young PH, Zamir S (ed) Handbooks of Game Theory with Economic Applications Vol.4. Elsevier, Amsterdam, pp 619-702.

15. Hamming RW (1950) Error detecting and error correcting codes. Bell Syst Tech J 29: 147-160.

16. Heifetz A, Samet D (1998) Topology-free typology of beliefs. J Econ Theory 82:324-341.

17. Myerson, RB (1978) Refinements of Nash equilibrium concept. Int J Game Theory 7:73-80.

18. Pearce D (1984) Rational strategic behavior and the problem of perfection. Econometrica 52:10291050 .

19. Perea A (2012) Epistemic Game Theory: Reasoning and Choice. Cambridge University Press, Cambridge.

20. Perea A, Kets W (2016) When do types induce the same belief hierarchy? Games 7. http://dx.doi.org/10.3390/g7040028

21. Perea A, Roy S (2017) A new epistemic characterization of $\varepsilon$-proper rationalizability. Games Econ Behav 104:309-328.

22. Samuelson L (1992) Dominated strategies and common knowledge. Games Econ Behav 4:284-313.

23. Schuhmacher F (1999) Proper rationalizability and backward induction. Int J Game Theory 28:599615.

24. Selten R (1975) Reexamination of the perfectness concept for equilibrium points in extensive games. Int J Game Theory 4:25-55. 